\title{
$\eta$-pairing states as true scars in an extended Hubbard model
}

\author{
Daniel K. Mark ๑ and Olexei I. Motrunich ๑ \\ Department of Physics, California Institute of Technology, Pasadena, California 91125, USA
}

(Received 8 June 2020; accepted 31 July 2020; published 24 August 2020)

\begin{abstract}
The $\eta$-pairing states are a set of exactly known eigenstates of the Hubbard model on hypercubic lattices, first discovered by Yang [C. N. Yang, Phys. Rev. Lett. 63, 2144 (1989)]. These states are not many-body scar states in the Hubbard model because they occupy unique symmetry sectors defined by the so-called $\eta$-pairing SU(2) symmetry. We study an extended Hubbard model with bond-charge interactions, popularized by Hirsch [J. E. Hirsch, Physica C 158, 326 (1989)], where the $\eta$-pairing states survive without the $\eta$-pairing symmetry and become true scar states. We also discuss similarities between the $\eta$-pairing states and exact scar towers in the spin-1 XY model found by Schecter and Iadecola [M. Schecter and T. Iadecola, Phys. Rev. Lett. 123, 147201 (2019)], and systematically arrive at all nearest-neighbor terms that preserve such scar towers in one dimension. We also generalize these terms to arbitrary bipartite lattices. Our study of the spin-1 XY model also leads us to several scarred models, including a spin-1/2 $J_{1}-J_{2}$ model with Dzyaloshinskii-Moriya interaction, in realistic quantum magnet settings in one and two dimensions.
\end{abstract}

DOI: 10.1103/PhysRevB.102.075132

\section{INTRODUCTION}

Quantum many-body scar states refer to sets of exceptional states in the spectra of some many-body nonintegrable models. These states do not obey the eigenstate thermalization hypothesis (ETH) [1,2], a framework used to describe how closed quantum many-body systems equilibrate to thermal distributions for local measurements. Scar states violate the ETH by having local quantities that are different from those of nearby states in energy, and in particular by having subvolume-law entanglement entropy (EE) scaling. Unlike manybody localized systems [3-11], where every eigenstate violates the ETH, quantum many-body scarred systems are an interesting class of models where only a small number of eigenstates violate the ETH in an otherwise thermal spectrum.

Quantum many-body scarring was first observed in a cold Rydberg atom experiment [12]. The experiment is modeled by the "PXP model" $[13,14]$. While there are several approximate ways of understanding the scar states in the PXP model [12-27], only some eigenstates in the middle of the spectrum are known exactly $[17,21]$.

Conversely, there are nonintegrable systems with exactly known scar states, such as the Affleck-Kennedy-Lieb-Tasaki (AKLT) model [28-30], the spin-1 XY model [31,32], and a spin-1/2 domain-wall-conserving model [33,34], among others [35-43], including a framework to make target states as scars in nonintegrable models $[35,36]$; there is also a growing number of examples of scars in the Floquet setting [41,4448].

The Hubbard model stands apart from this list of models. There are exactly known states embedded in the spectrumthe $\eta$-pairing states due to Yang [49]. These $\eta$-pairing states are known to have sub-volume-law entanglement [50]. However, they do not constitute many-body scar states because the Hubbard model possesses an additional SU(2) symmetry [51], which we refer to as the " $\eta$-pairing SU(2)." The $\eta$ - pairing states are the unique eigenstates in the symmetry sector of maximal $\eta$-pairing SU(2) total spin and are therefore not expected to be thermalized with respect to the rest of the spectrum. While there are several proposals for unusual thermalization in the Hubbard model [52-56], in this paper we discuss a direct way to make an electronic Hubbard-like model quantum scarred by the $\eta$-pairing states.

We will present several terms that break the $\eta$-pairing $\mathrm{SU}(2)$ symmetry, but preserve the $\eta$-pairing states $\left|\psi_{N}\right\rangle$ (defined below) as eigenstates. This can be achieved while retaining the spin $\mathrm{SU}(2)$ symmetry, so $\left|\psi_{N}\right\rangle$ will be eigenstates in the otherwise thermal sector with quantum numbers total spin $S=0$, momentum $k=N \pi(\bmod 2 \pi)$, site inversion $I_{s}=1$, and fermion species numbers $N_{\uparrow}=N_{\downarrow}=N$, and thus constitute many-body scar states. The most notable of these perturbations is the Hirsch model discussed below.

In Sec. II we review the Hubbard model and the Hirsch model, and discuss how the $\eta$-pairing states (and some related states) are scars in this model. In order to discuss our systematic construction of terms that make the $\eta$-pairing states scars, in Sec. III we first draw parallels between spin-1 and electronic models, in particular between the spin-1 XY model scars and the $\eta$-pairing states. In Sec. IV we systematically construct such scarred models in spin-1 systems, which goes beyond previously known models. We then map these results over to electronic systems in Sec. V, with the result that the Hirsch model belongs to a small family of models scarred by the $\eta$-pairing states, and is arguably the most easily realized of this family.

Lastly, our study of the spin-1 XY model scars with $k=\pi$ bimagnon towers naturally leads us to consider $k=0$ bimagnon towers as scars. In Appendix D, we show that the spin-1 $k=0$ bimagnon towers are scars in a model with the Dzyaloshinskii-Moriya interaction (DMI) replacing the $\mathrm{XY}$ spin-exchange term. In the presence of conservation of the number of zeros (i.e., sites where $S_{j}^{z}=0$ ), the $k=0$ 
bimagnon tower maps onto a spin-1/2 $k=0$ magnon tower. We also find a model scarred by this tower: the $J_{1}-J_{2}$ model with spin-1/2 DMI.

\section{HIRSCH MODEL: THE HUBBARD MODEL WITH BOND-CHARGE INTERACTIONS}

\section{A. Hubbard model, $\eta$-pairing states, and $\eta$-pairing $\mathrm{SU}(2)$ symmetry}

The Hubbard model is a model of interacting electrons given by

$$
\begin{aligned}
H_{\mathrm{Hub}}= & -t \sum_{\langle\mathbf{i j}\rangle, \sigma}\left(c_{\mathbf{i}, \sigma}^{\dagger} c_{\mathbf{j}, \sigma}+\text { H.c. }\right)+U \sum_{\mathbf{j}} n_{\mathbf{j}, \uparrow} n_{\mathbf{j}, \downarrow} \\
& -\mu \sum_{\mathbf{j}, \sigma} n_{\mathbf{j}, \sigma},
\end{aligned}
$$

where $c_{\mathbf{j}, \sigma}^{\dagger}$ creates an electron at site $\mathbf{j}$ with spin $\sigma$, and $n_{\mathbf{j}, \sigma}=$ $c_{\mathbf{j}, \sigma}^{\dagger} c_{\mathbf{j}, \sigma}$. The model is composed of hopping, interaction, and chemical potential terms with coefficients $t, U$, and $\mu$, respectively. For analysis in later sections, it will be convenient to denote the on-site states as $\{h, \uparrow, \downarrow, d\}$, where $h$ indicates an empty site ("holon"), $\uparrow$ or $\downarrow$ indicates a singly occupied site with a spin-up or spin-down electron, and $d$ indicates a doubly occupied site ("doublon"). The Hubbard model on the one-dimensional (1D) chain with nearest-neighbor hopping is exactly solved [57], but this is not important for the present paper.

Yang [49] introduced the $\eta$-pairing states as eigenstates of the Hubbard model on any bipartite lattice. For our purposes we will specialize to hypercubic lattices of $V$ sites. The $\eta$ pairing states are

$$
\left.\left|\psi_{N}\right\rangle=C_{N}\left(\eta^{\dagger}\right)^{N} \mid \text { vac. }\right\rangle
$$

where

$$
\eta^{\dagger}=\sum_{\mathbf{j}} e^{i \mathbf{r}_{\mathbf{j}} \cdot \pi} c_{\mathbf{j}, \uparrow}^{\dagger} c_{\mathbf{j}, \downarrow}^{\dagger},
$$

where $C_{N}=\sqrt{(V-N) ! /(V ! N !)}$ is the normalization constant, $\mid$ vac. $\rangle$ is the electronic vacuum state, and $\pi=$ $(\pi, \pi, \ldots, \pi)$. The number of pairs $N$ can range from zero to $V$. We also note that our definition of $\eta^{\dagger}$ differs from that in the literature by a sign - this choice is made for easy comparison with the spin-1 model operator $Q^{\dagger}$ below. There is a significant body of work investigating the possibility of realizing these states as ground states as well as signatures of $\eta$ pairing in Hubbard models (see, e.g., Refs. [58-63]).

$\left|\psi_{N}\right\rangle$ has energy $(U-2 \mu) N$. It is trivially an eigenstate of the interaction and chemical potential terms, and is annihilated by the hopping term, owing to cancellations from the momentum $\pi$ construction [49].

When $U=2 \mu, H_{\mathrm{Hub}}$ in fact is $\mathrm{SU}(2)$ symmetric under the following generators [51]: $\left[H_{\mathrm{Hub}}, \eta^{\dagger}\right]=\left[H_{\mathrm{Hub}}, \eta\right]=$ $\left[H_{\mathrm{Hub}}, \eta^{z}\right]=0$, where

$$
\eta^{z}=\frac{1}{2}\left(\sum_{\mathbf{j}, \sigma} n_{\mathbf{j}, \sigma}-V\right)=\frac{1}{2}\left(N_{\uparrow}+N_{\downarrow}-V\right) .
$$

$H_{\mathrm{Hub}}$ is also $\mathrm{SU}(2)$ symmetric under the total electron-spin generators $S^{ \pm}, S^{z}$, where

$$
\begin{aligned}
S^{+} & =\sum_{\mathbf{j}} c_{\mathbf{j}, \uparrow}^{\dagger} c_{\mathbf{j}, \downarrow}, \quad S^{-}=\left(S^{+}\right)^{\dagger}, \\
S^{z} & =\frac{1}{2} \sum_{\mathbf{j}}\left(n_{\mathbf{j}, \uparrow}-n_{\mathbf{j}, \downarrow}\right)=\frac{1}{2}\left(N_{\uparrow}-N_{\downarrow}\right) .
\end{aligned}
$$

The generators $\eta^{\dagger}, \eta, \eta^{z}$ and $S^{ \pm}, S^{z}$ obey SU(2) commutation relations $\left[\eta^{z}, \eta^{\dagger}\right]=\eta^{\dagger},\left[\eta^{\dagger}, \eta\right]=2 \eta^{z}$, and all $\eta$-pairing generators commute with all spin SU(2) generators. Therefore there are two independent $\mathrm{SU}(2)$ symmetries in $H_{\mathrm{Hub}}$. Changing $\mu$ simply shifts the energies by the corresponding values determined by the $\eta^{z}$ quantum numbers and does not affect the eigenstates or change any symmetry sectors.

The $\eta$-pairing states $\left|\psi_{N}\right\rangle$ lie in the spin sector $S=0$. They have $\eta^{z}=(2 N-V) / 2$. Therefore, as $N$ values run from zero to $V$, they comprise the unique multiplet of states with the maximum possible total $\eta$-pairing "spin" $V / 2$.

On any lattice, at fixed pair density $v=N / V$, these states were shown in Ref. [50] to have sub-volume-law EE:

$$
S_{A}=\frac{1}{2}\left\{1+\ln \left[2 \pi v(1-v) V_{A}\right]\right\},
$$

where $S_{A}$ is measured at a cut which partitions $V$ into subsystems $A$ with $V_{A}$ sites and $B$ with $V-V_{A}$ sites. The EE of an eigenstate $|\psi\rangle$ is given by $S_{A}=-\operatorname{Tr}\left(\rho_{A} \log \rho_{A}\right)$, where $\rho_{A}=$ $\operatorname{Tr}_{B}(|\psi\rangle\langle\psi|)$ is the reduced density matrix of that eigenstate with respect to subsystem $A$.

However, because $\left|\psi_{N}\right\rangle$ are the unique states in a given $\eta^{z}$, $\eta^{2}$ sector, Ref. [50] concluded that they did not violate the ETH.

Lastly, we note that other related exact states are known in the Hubbard model [50,51]:

$$
\left.\left|\psi_{N}^{\{\mathbf{k}\}}\right\rangle=C_{N}^{\{\mathbf{k}\}}\left(\eta^{\dagger}\right)^{N} \prod_{\mathbf{k} \in \mathcal{F}} c_{\downarrow}^{\dagger}(\mathbf{k}) \mid \text { vac. }\right\rangle,
$$

for any set $\mathcal{F}$ of wave vectors $\mathbf{k}$, because there is no interaction between electrons of the same spin species. However, we restrict our attention to the original $\eta$-pairing states $\left|\psi_{N}\right\rangle$ because they are the only states that survive under the Hirsch term and other perturbations (and in any dimension).

\section{B. Hirsch model}

The Hirsch model was popularized by Hirsch [64] and Hirsch and Marsiglio [65] as a model of hole superconductivity. It is given by

$$
\begin{aligned}
H= & -\sum_{\langle\mathbf{i j}\rangle, \sigma}\left[t-X\left(n_{\mathbf{i},-\sigma}+n_{\mathbf{j},-\sigma}\right)\right]\left(c_{\mathbf{i}, \sigma}^{\dagger} c_{\mathbf{j}, \sigma}+\text { H.c. }\right) \\
& +U \sum_{\mathbf{j}} n_{\mathbf{j}, \uparrow} n_{\mathbf{j}, \downarrow}-\mu \sum_{\mathbf{j}, \sigma} n_{\mathbf{j}, \sigma}=H_{\text {Hub }}+H_{\text {Hirsch }},
\end{aligned}
$$

where " $-\sigma$ " indicates the opposite spin species to $\sigma$. The Hirsch model adds to the Hubbard model a "bond-charge interaction" term [59,66-69], which modifies the hopping constant depending on the occupations across the bond. This term is alternatively called "correlated hopping" [70,71]. This term was originally estimated by Hubbard [72] in solid-state 

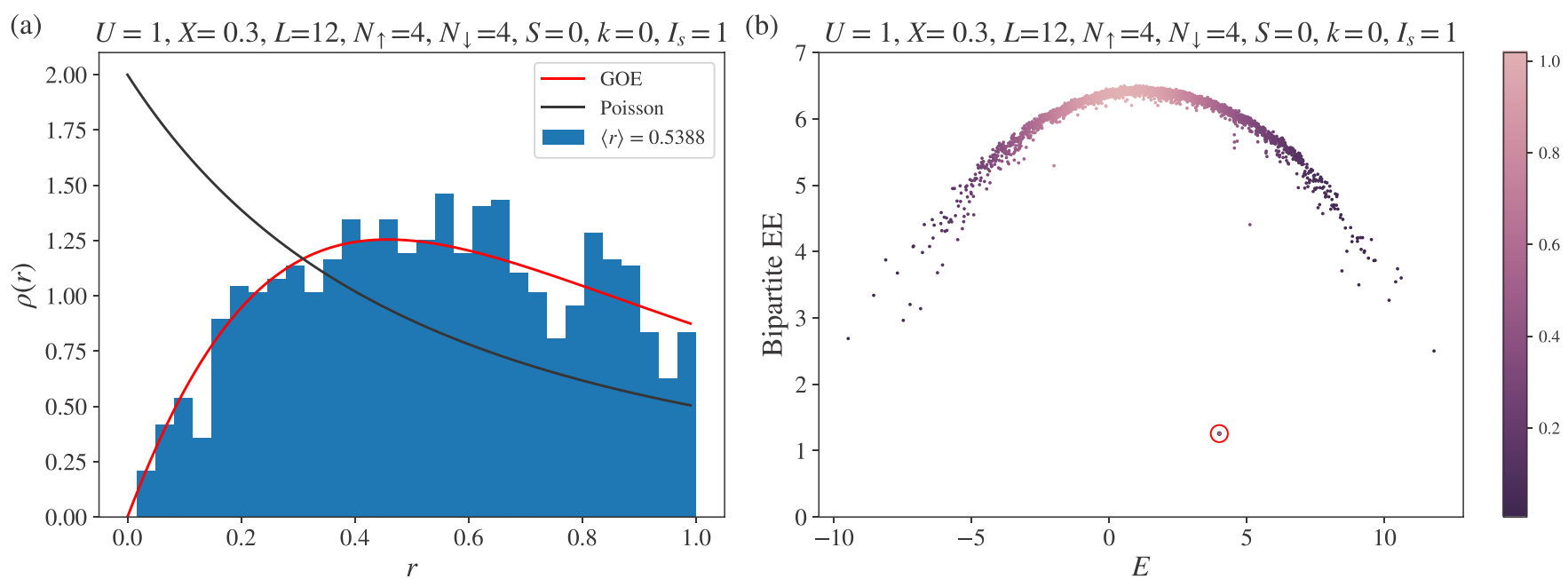

FIG. 1. (a) $r$ statistics of the Hirsch model with $U=t=1$ and $X=0.3$ on a periodic chain of length $L=12$, in the symmetry sector $N_{\uparrow}=N_{\downarrow}=4$, total spin $S=0$, momentum $k=0$, and site inversion $I_{s}=1$. The Hilbert-space dimension is 3072. The $r$ statistics are consistent with the Wigner-Dyson GOE prediction for quantum chaotic models. (b) Bipartite EE in the same symmetry sector. The $\eta$-pairing state $\left|\psi_{4}\right\rangle$ is a clear EE outlier. Each point is colored by the density of states at the corresponding energy, with colorbar (normalized to 1) on the right.

systems, and there are also proposals to realize this with ultracold atoms [73]. We lastly note that the 1D Hirsch model is integrable when $X=t[74,75]$, but this special point is not important for the scar physics of interest to us.

The Hirsch term breaks the $\eta$-pairing $\mathrm{SU}(2)$ symmetry, which can be verified by evaluating the commutator $\left[H_{\text {Hirsch }}, \eta^{\dagger}\right]$. However, the Hirsch term preserves the spin $\mathrm{SU}(2)$. This is clear by the rewriting on each bond $\langle\mathbf{i j}\rangle$ :

$$
\begin{aligned}
& \sum_{\sigma}\left(n_{\mathbf{i},-\sigma}+n_{\mathbf{j},-\sigma}\right)\left(c_{\mathbf{i}, \sigma}^{\dagger} c_{\mathbf{j}, \sigma}+\text { H.c. }\right) \\
& =\left[\sum_{\sigma^{\prime}}\left(n_{\mathbf{i}, \sigma^{\prime}}+n_{\mathbf{j}, \sigma^{\prime}}\right)-1\right] \sum_{\sigma}\left(c_{\mathbf{i}, \sigma}^{\dagger} c_{\mathbf{j}, \sigma}+\text { H.c. }\right) .
\end{aligned}
$$

From Eq. (10), we also immediately see that $H_{\text {Hirsch }}\left|\psi_{N}\right\rangle=$ 0 , because on bonds connecting opposite sublattices the rightmost operator in Eq. (10) annihilates $\left|\psi_{N}\right\rangle$ :

$\sum_{\sigma}\left(c_{\mathbf{i}, \sigma}^{\dagger} c_{\mathbf{j}, \sigma}+\right.$ H.c. $)\left|\psi_{N}\right\rangle=0$. The latter is the property from Yang's original paper [49] and is easily verified because the only nontrivial term is

$$
\begin{aligned}
& \left.\sum_{\sigma}\left(c_{\mathbf{i}, \sigma}^{\dagger} c_{\mathbf{j}, \sigma}+\text { H.c. }\right)\left(c_{\mathbf{i}, \uparrow}^{\dagger} c_{\mathbf{i}, \downarrow}^{\dagger}-c_{\mathbf{j}, \uparrow}^{\dagger} c_{\mathbf{j}, \downarrow}^{\dagger}\right) \mid \text { vac. }\right\rangle \\
& \left.\quad=\left(c_{\mathbf{j}, \uparrow}^{\dagger} c_{\mathbf{i}, \downarrow}^{\dagger}-c_{\mathbf{j}, \downarrow}^{\dagger} c_{\mathbf{i}, \uparrow}^{\dagger}-c_{\mathbf{i}, \uparrow}^{\dagger} c_{\mathbf{j}, \downarrow}^{\dagger}+c_{\mathbf{i}, \downarrow}^{\dagger} c_{\mathbf{j}, \uparrow}^{\dagger}\right) \mid \text { vac. }\right\rangle=0 .
\end{aligned}
$$

Therefore the $\eta$-pairing states $\left|\psi_{N}\right\rangle$ remain eigenstates of $H$ for any strength of the Hirsch term. They are embedded in the symmetry sectors $S=0, \mathbf{k}=N \pi(\bmod 2 \pi), I_{s}=1, N_{\uparrow}=$ $N_{\downarrow}=N$, where $S$ denotes total spin, $\mathbf{k}$ momentum, $I_{s}$ site inversion, and $N_{\sigma}$ the number of $\sigma$ species electrons.

From numerical studies of the above model in 1D systems with periodic boundary conditions ( $\mathrm{PBCs}$ ), we verify that the level-spacing statistics in these sectors follow the WignerDyson Gaussian orthogonal ensemble (GOE) predictions [see
Fig. 1(a)] and that $\left|\psi_{N}\right\rangle$ are bipartite EE outliers in their respective sectors [see Fig. 1(b)].

To compute the $r$ statistics, we take the spacing between consecutive energies: $s_{n}=E_{n+1}-E_{n}$. The ratio $r_{n}$ is given by $r_{n}=\min \left(s_{n} / s_{n-1}, s_{n-1} / s_{n}\right)$ and is a frequently used diagnostic of many-body nonintegrability $[4,76] . r_{n}$ is bounded between 0 and 1, and we plot its histogram distribution in Fig. 1(a). The bipartite EE is defined as the entanglement entropy of a cut in the middle of a chain of length $L$, i.e., that divides the chain into subsystems $A=\{1, \ldots, L / 2\}$ and $B=\{L / 2+1, \ldots, L\}$.

We therefore conclude that in the Hirsch model the $\eta$ pairing states constitute true many-body scar states. We finally note that while the Hirsch model has been around for some time the presence of the scar states in the nonintegrable model was not known. Also, we identified it through a systematic study of two-site terms that break the $\eta$-pairing $\mathrm{SU}(2)$ but preserve the $\eta$-pairing states, discussed in Sec. V below.

Lastly, while we identified the Hirsch model as a model that contains the $\eta$-pairing states as scars, our subsequent numerical investigation revealed additional entanglement outlier states in one dimension, some of which constitute two additional scar towers $\left|\psi_{N, M}\right\rangle=\left(t^{\dagger}\right)^{M}\left|\psi_{N}\right\rangle$ and $\left|\phi_{N}\right\rangle=s^{\dagger}\left|\psi_{N}\right\rangle$. The operators $t^{\dagger}$ and $s^{\dagger}$ create nearest-neighbor triplets $|\uparrow, \uparrow\rangle$ and singlets $|\uparrow, \downarrow\rangle-|\downarrow, \uparrow\rangle$, with momentum $\pi$. They are defined in Appendix A, in which we also prove these states.

\section{ANALOGY BETWEEN THE SPIN-1 XY SCAR TOWER AND $\eta$-PAIRING STATES}

To systematically construct Hamiltonians that make the $\eta$-pairing states scarry, we first understand scarring in spin-1 systems. In particular, the spin-1 XY model $[31,32]$ is known to contain an exact tower of scar states, which are analogous to the $\eta$-pairing states in the Hubbard model. We first construct scarred models in the simpler spin-1 setting, then show how these results translate to electronic models. We also note that while there is a separate tower of scar states in the spin-1 
AKLT model $[28,29]$ their electronic model analogs are not immediate.

The spin-1 XY model is one of the simplest models known to have an exact tower of scar states [31,32]. In Ref. [31], Schecter and Iadecola considered spin-1 XY models of the form

$$
\begin{aligned}
H_{\text {Spin- } 1} & =J \sum_{\langle\mathbf{i} \mathbf{j}\rangle}\left(S_{\mathbf{i}}^{x} S_{\mathbf{j}}^{x}+S_{\mathbf{i}}^{y} S_{\mathbf{j}}^{y}\right)+h \sum_{\mathbf{j}} S_{\mathbf{j}}^{z}+D \sum_{\mathbf{j}}\left(S_{\mathbf{j}}^{z}\right)^{2} \\
& =H_{\mathrm{XY}}+H_{z}+H_{z^{2}} .
\end{aligned}
$$

Here the $\mathbf{S}_{\mathbf{i}}$ are spin-1 spin operators. In what follows whether the $S$ 's refer to spin operators of the spin-1 model or electronspin operators of the Hubbard model should be clear from context.

In one dimension, one has to introduce a third-neighbor term $H_{3}=J_{3} \sum_{j}\left(S_{j}^{x} S_{j+3}^{x}+S_{j}^{y} S_{j+3}^{y}\right)$ to break a special nonlocal SU(2) symmetry present in sectors with even magnetization $[32,77]$. On hypercubic lattices, the scar tower $\left|\mathcal{S}_{N}\right\rangle$ is formed by the operator $Q^{\dagger}$ :

$$
\left|\mathcal{S}_{N}\right\rangle=\left(Q^{\dagger}\right)^{N}|\Omega\rangle, \quad Q^{\dagger}=\frac{1}{2} \sum_{\mathbf{j}} e^{i \mathbf{r}_{\mathbf{j}} \cdot \pi}\left(S_{\mathbf{j}}^{+}\right)^{2},
$$

where $|\Omega\rangle=|-1,-1, \ldots,-1\rangle$ and $N=0, \ldots, V$. These states have energies $E_{N}=h(2 N-V)+D V$.

We immediately see that the $Q^{\dagger}$ operator in the spin-1 model is analogous to the $\eta^{\dagger}$ operator in the Hubbard model [Eq. (3)]. $\frac{1}{2}\left(S^{+}\right)^{2}$ sends $|-1\rangle \rightarrow|1\rangle$, while $c_{\uparrow}^{\dagger} c_{\downarrow}^{\dagger}$ sends $|h\rangle \rightarrow$ $|d\rangle$, where $h$ indicates an empty site ("holon") and $d$ indicates a doubly occupied site ("doublon"). This hints at identifying the spin-1 states $|-1\rangle,|1\rangle$ with the electronic $|h\rangle,|d\rangle$, respectively. Furthermore, comparison of $\mathrm{SU}(2)$ algebra relations such as $\left[Q^{\dagger}, Q\right]=S_{\mathrm{Tot}}^{z}$ in the spin-1 case with $\left[\eta^{\dagger}, \eta\right]=N_{\uparrow}+$ $N_{\downarrow}-V$ in the electron case suggests relating spin-1 models with $S_{\mathrm{Tot}}^{z}$ conservation and electronic models with conserved total electron number. There is some ambiguity with identification of the spin- 1 state $|0\rangle$, but we will argue that for the purposes of our paper electron-spin SU(2) invariance-which is natural to require in an electronic model to reduce the space of models under consideration-makes our identification unambiguous.

\section{SYSTEMATIC CONSTRUCTION OF SPIN-1 MODELS WITH THE XY SCAR TOWER}

In Ref. [31], Schecter and Iadecola noted an SU(2) symmetry obeyed only by the scar tower $\left|\mathcal{S}_{N}\right\rangle$. In Ref. [34], we found a model "embedded" in the XY model:

$$
H_{0}=\sum_{\langle\mathbf{i j}\rangle}(|1,0\rangle\langle 0,1|-|-1,0\rangle\langle 0,-1|+\text { H.c. })_{\mathbf{i}, \mathbf{j}},
$$

such that

$$
H_{\mathrm{XY}}=H_{0}+H_{\mathrm{XY}}^{\prime},
$$

where

$$
\begin{aligned}
H_{\mathrm{XY}}^{\prime}= & \sum_{\langle\mathbf{i j}\rangle}[(|1,-1\rangle+|-1,1\rangle)\langle 0,0| \\
& +2|-1,0\rangle\langle 0,-1|+\text { H.c. }]_{\mathbf{i}, \mathbf{j}} .
\end{aligned}
$$

This rewriting is significant because $H_{0}$ commutes with $Q^{\dagger}$ and $S_{\mathrm{Tot}}^{z}$, while $H_{\mathrm{XY}}^{\prime}$ annihilates $\left|\mathcal{S}_{N}\right\rangle$. Operators $Q^{\dagger}, Q$, and $Q^{z} \equiv S_{\text {Tot }}^{z} / 2$ obey the $\mathrm{SU}(2)$ commutation relations and therefore generate a "pseudospin" SU(2) symmetry present in $H_{0}$ analogous to the $\eta$-pairing $\mathrm{SU}(2)$ in the Hubbard model. As with the $\eta$-pairing states, $\left|\mathcal{S}_{N}\right\rangle$ are the unique states occupying the sector of the highest total pseudospin $V / 2$. The pseudospin $\mathrm{SU}(2)$ is broken by $H_{\mathrm{XY}}^{\prime}$, and hence the total Hamiltonian $H_{\mathrm{XY}}$ contains $\left|\mathcal{S}_{N}\right\rangle$ in an otherwise thermal spectrum.

The above observation inspired this paper, and a natural question is to systematically generate all other spin- 1 nearestneighbor models that share the scar tower $\left|\mathcal{S}_{N}\right\rangle$. To do so we list the following operators: (A) nearest-neighbor operators that commute with $Q^{\dagger}$ and $S_{\mathrm{Tot}}^{z}$, (B) nearest-neighbor operators $O$ such that $\left[O, Q^{\dagger}\right]=Q^{\dagger}$, and (C) nearest-neighbor operators that annihilate $\left|\mathcal{S}_{N}\right\rangle$. For simplicity, in each group we only consider Hermitian operators, although our procedure is also able to find non-Hermitian operators satisfying the above properties.

It is clear that if $|\Omega\rangle$ is an eigenstate of a model in group A so too are all the $\left|\mathcal{S}_{N}\right\rangle$. This is immediately true for the state $|\Omega\rangle$, because this state has the highest possible pseudospin quantum number of $V / 2$ and hence is always an eigenstate of any model with the corresponding $\mathrm{SU}(2)$ symmetry.

It is natural to consider terms in groups B and C modulo those in group A. As discussed below, it turns out that the unique operator in group B, modulo terms in group A, is $Q^{z}=S_{\mathrm{Tot}}^{z} / 2$. Adding a term proportional to $Q^{z}$ to the Hamiltonian uniformly shifts each $S_{\text {Tot }}^{z}$ sector in energy, but does not change any eigenvectors or symmetry sectors. In any model composed of terms from groups $\mathrm{A}$ and $\mathrm{B}$, the Schecter-Iadecola states $\left|\mathcal{S}_{N}\right\rangle$ will not be "true" scar states for the same reasons that the $\eta$-pairing states are not in the original Hubbard model.

We can break the pseudospin symmetry and turn $\left|\mathcal{S}_{N}\right\rangle$ into true scars with any linear combination of such a model and those in group $\mathrm{C}$ that annihilate $\left|\mathcal{S}_{N}\right\rangle$. In the example in Eq. (15), $H_{0}$ belongs to group $\mathrm{A}$, and $H_{\mathrm{XY}}^{\prime}$ belongs to group $\mathrm{C}$. The energies of the scar states are split by the term $H_{z}=h S_{\mathrm{Tot}}^{z}$.

\section{A. Operators that commute with $Q^{\dagger}$ and $S_{\text {Tot }}^{z}$}

We first note that $\left[O, S_{\mathrm{Tot}}^{z}\right]=0$ is a necessary condition for $\left[O, Q^{\dagger}\right]=0$, because $S_{\mathrm{Tot}}^{z}=\left[Q^{\dagger}, Q\right]$.

It is immediate that the only on-site operators that commute with $Q^{\dagger}$ are $|0\rangle\left\langle\left. 0\right|_{j}\right.$ and $\left.\mid 1\right\rangle\left\langle\left. 1\right|_{j}+\mid-1\right\rangle\left\langle-\left.1\right|_{j}=\left(S_{j}^{z}\right)^{2}\right.$ (the two terms sum to identity, and so are not independent for our purposes). We list these operators in Table I for reference. We also list their translations to electronic models, which will be explained later in Sec. V.

We then focus our attention on two-site (nearest-neighbor) operators. The results in group A below hold on any bipartite lattice. However, because we will rely on matrix product state (MPS) techniques to find operators that annihilate $\left|\mathcal{S}_{N}\right\rangle$, we will simplify our notation to the 1D case going forward.

By elementary methods discussed in Appendix B, we find that terms $1-7$ in Table II commute with $Q^{\dagger}$.

We recover known terms that commute with $Q^{\dagger}$, such as $H_{0}$ in Eq. (14) (no. 5 with $\phi=0$ ) and the pure biquadratic term $\left(\mathbf{S}_{j} \cdot \mathbf{S}_{j+1}\right)^{2}$ [30] [no. 1-no. $6(\phi=0)+$ no. 
TABLE I. On-site operators that either commute with $Q^{\dagger}$ or satisfy $\left[O, Q^{\dagger}\right]=Q^{\dagger}$. The two on-site operators in group A sum to identity.

\begin{tabular}{cc}
\hline \hline Spin-1 model & Electronic model \\
\hline A. Operators that commute with $Q^{\dagger}$, or with $\eta^{\dagger}$ \\
$|0\rangle\left\langle\left. 0\right|_{j}\right.$ & $|\uparrow\rangle\left\langle\left.\uparrow\right|_{j}+\mid \downarrow\right\rangle\left\langle\left.\downarrow\right|_{j}\right.$ \\
$|1\rangle\left\langle\left. 1\right|_{j}+\mid-1\right\rangle\left\langle-\left.1\right|_{j}\right.$ & $|d\rangle\left\langle\left. d\right|_{j}+\mid h\right\rangle\left\langle\left. h\right|_{j}\right.$ \\
B. Operators $O$ with $\left[O, Q^{\dagger}\right]=Q^{\dagger}$, or $\left[O, \eta^{\dagger}\right]=\eta^{\dagger}$ \\
$\frac{1}{2} \sum_{j} S_{j}^{z}=Q^{z}$ & $\frac{1}{2} \sum_{j}\left(|d\rangle\left\langle\left. d\right|_{j}-\mid h\right\rangle\left\langle\left. h\right|_{j}\right)=\eta^{z}\right.$ \\
\hline \hline
\end{tabular}

$7+I]$. We lastly note that there are longer-range terms that commute with $Q^{\dagger}$, such as $\sum_{j}|0,0,0\rangle\left\langle 0,0,\left.0\right|_{j, j+1, j+2}-\right.$ one could also systematically construct them with such an approach.

\section{B. Operators with $\left[O, Q^{\dagger}\right]=Q^{\dagger}$}

The computation in the previous section also immediately gives us that the only nearest-neighbor operator that satisfies $\left[O, Q^{\dagger}\right]=Q^{\dagger}$ is in fact the on-site operator $Q^{z}=S_{\mathrm{Tot}}^{z} / 2$ (no. 8 ), modulo any linear combination of operators in group $\mathrm{A}$ (nos. $1-7$ in Table II). We can prove this fact: given an $O$ such that $\left[O, Q^{\dagger}\right]=Q^{\dagger}$, we write $O=O-Q^{z}+Q^{z}$. It follows that $\left[O-Q^{z}, Q^{\dagger}\right]=0$, so $O$ will be the sum of terms in group A and $Q^{z}$. Therefore $Q^{z}$ is the only independent term in group B.

\section{Operators that annihilate the scar tower}

We next study nearest-neighbor operators that annihilate the scar tower $\left|\mathcal{S}_{N}\right\rangle$. To do so we compress all $\left|\mathcal{S}_{N}\right\rangle$ into a single MPS: $|\mathcal{S}(z)\rangle=\sum_{N}\left(z^{N} / N !\right)\left|\mathcal{S}_{N}\right\rangle=\exp \left(z Q^{\dagger}\right)|\Omega\rangle$, with parameter $z$. We then express nearest-neighbor operators as matrix product operators (MPOs). Focusing on operators that conserve $S_{\text {Tot }}^{z}$, it suffices to find all such nearest-neighbor operators that annihilate $|\mathcal{S}(z)\rangle$. We adopt an approach similar to Ref. [78]. In the MPO and MPS language this becomes a problem of finding all null eigenvectors of some small matrix [Eq. (28) below]. The parameter $z$ is arbitrary, and for our numerical computations we take $z=0.1$. Before proceeding with details, we note that in the present case $|\mathcal{S}(z)\rangle$ is a product state, and we could in principle calculate without using the full MPO-MPS formalism (and even find all scarry models without compressing the scar tower, see Appendix C). Nevertheless, the presented formalism is very powerful and can be applied as a black-box tool to any set of scars compressed into an MPS and will be applied also to the AKLT scar tower in Appendix F.

Writing $\exp \left(z Q^{\dagger}\right)$ in MPO form,

$$
\begin{aligned}
\exp \left(z Q^{\dagger}\right) & =b_{l}^{T}\left(\prod_{j=1}^{L} M_{j}\right) b_{r}, \\
M_{j} & =\left(\begin{array}{cc}
-\mathbf{I}_{j} & -z\left(S_{j}^{+}\right)^{2} / 2 \\
z\left(S_{j}^{+}\right)^{2} / 2 & \mathbf{I}_{j}
\end{array}\right), \\
b_{l} & =\left(\begin{array}{l}
1 \\
0
\end{array}\right), \quad b_{r}=\left(\begin{array}{l}
1 \\
1
\end{array}\right),
\end{aligned}
$$

TABLE II. Nearest-neighbor operators that either commute with $Q^{\dagger}$, satisfy $\left[O, Q^{\dagger}\right]=Q^{\dagger}$, or annihilate the scar states $\left|\mathcal{S}_{N}\right\rangle$. In the left column, we show Hermitian operators in the spin-1 model that preserve $S_{\mathrm{Tot}}^{z}$, while in the right column we show translations to the electronic model in the ket-bra notation (see Sec. V). Operators 1-4 sum to the identity, and the on-site operators in Table I are linear combinations of nos. 1-4, e.g., $|0\rangle\left\langle\left. 0\right|_{j}=\right.$ no. $1+$ no. 4 . We also note that in nos. 9-11 the +1 and -1 options are not independent - they are related by operators 3-5 in group A. While the systematic procedure for finding group C assumes translationally invariant operators on the 1D chain, we drop the summation over $j$ where it is not necessary: nos. 1-11 can be generalized to any bipartite lattice.

\begin{tabular}{|c|c|c|}
\hline 1 & $|00\rangle\left\langle\left. 00\right|_{j, j+1}\right.$ & $\left|\sigma_{1}, \sigma_{2}\right\rangle\left\langle\sigma_{3},\left.\sigma_{4}\right|_{j, j+1}+\right.$ H.c., $\sigma_{1}+\sigma_{2}=\sigma_{3}+\sigma_{4}$ \\
\hline 2 & $(|1\rangle\langle 1|+|-1\rangle\langle-1|)_{j}(|1\rangle\langle 1|+|-1\rangle\langle-1|)_{j+1}$ & $(|d\rangle\langle d|+| h\rangle\langle h|)_{j}(|d\rangle\langle d|+| h\rangle\langle h|)_{j+1}$ \\
\hline 3 & $(|1\rangle\langle 1|+|-1\rangle\langle-1|)_{j}(|0\rangle\langle 0|)_{j+1}$ & $(|d\rangle\langle d|+| h\rangle\langle h|)_{j}(|\uparrow\rangle\langle\uparrow|+| \downarrow\rangle\langle\downarrow|)_{j+1}$ \\
\hline 4 & $(|0\rangle\langle 0|)_{j}(|1\rangle\langle 1|+|-1\rangle\langle-1|)_{j+1}$ & $(|\uparrow\rangle\langle\uparrow|+| \downarrow\rangle\langle\downarrow|)_{j}(|d\rangle\langle d|+| h\rangle\langle h|)_{j+1}$ \\
\hline 5 & $e^{i \phi}(|0,1\rangle\langle 0,1|-| 0,-1\rangle\langle-1,0|)_{j, j+1}+$ H.c. & $\begin{array}{l}e^{i \phi}(|\uparrow, d\rangle\langle d, \uparrow|+| \downarrow, d\rangle\langle d, \downarrow|-| \uparrow, h\rangle\langle h, \uparrow| \\
\quad-|\downarrow, h\rangle\langle h, \downarrow|)_{j, j+1}+\text { H.c. }\end{array}$ \\
\hline 6 & $e^{i \phi}|00\rangle(\langle 1,-1|+\langle-1,1|)_{j, j+1}+$ H.c. & $e^{i \phi}(|\uparrow, \downarrow\rangle-|\downarrow, \uparrow\rangle)(\langle d, h|+\langle h, d|)_{j, j+1}+$ H.c. \\
\hline \multicolumn{3}{|c|}{ B. Operators $O$ with $\left[O, Q^{\dagger}\right]=Q^{\dagger}$, or $\left[O, \eta^{\dagger}\right]=\eta^{\dagger}$} \\
\hline 8 & $Q^{z}=\frac{1}{2} \sum_{j} S_{j}^{z}$ & $\eta^{z}=\frac{1}{2} \sum_{j}\left(|d\rangle\left\langle\left. d\right|_{j}-\mid h\right\rangle\left\langle\left. h\right|_{j}\right)\right.$ \\
\hline \multicolumn{3}{|c|}{ C. Operators that annihilate scar tower } \\
\hline 9 & $| \pm 1,0\rangle\left\langle \pm 1,\left.0\right|_{j, j+1}\right.$ & $|d\rangle\left\langle\left. d\right|_{j}(|\uparrow\rangle\langle\uparrow|+| \downarrow\rangle\langle\downarrow|)_{j+1}\left(\right.\right.$ or $|h\rangle\left\langle\left. h\right|_{j}\right)$ \\
\hline 10 & $|0, \pm 1\rangle\left\langle 0, \pm\left. 1\right|_{j, j+1}\right.$ & $(|\uparrow\rangle\langle\uparrow|+| \downarrow\rangle\langle\downarrow|)_{j}|d\rangle\left\langle\left. d\right|_{j+1}\left(\right.\right.$ or $|h\rangle\left\langle\left. h\right|_{j+1}\right)$ \\
\hline
\end{tabular}

\begin{tabular}{lll}
\hline \hline No. & Spin-1 model operator & Electronic model operator \\
\hline
\end{tabular}

A. Operators that commute with $Q^{\dagger}$, or $\eta^{\dagger}$ 
we can write $|\mathcal{S}(z)\rangle$ in MPS form:

$$
\begin{aligned}
|\mathcal{S}(z)\rangle & =\sum_{\left\{\sigma_{j}\right\}} b_{l}^{T}\left(\prod_{j=1}^{L} A^{\left[\sigma_{j}\right]}\right) b_{r}\left|\left\{\sigma_{j}\right\}\right\rangle, \\
A^{[-1]} & =\left(\begin{array}{cc}
-1 & 0 \\
0 & 1
\end{array}\right), A^{[0]}=\mathbf{0}, \\
A^{[1]} & =z\left(\begin{array}{cc}
0 & -1 \\
1 & 0
\end{array}\right) .
\end{aligned}
$$

We express translationally invariant operators $O$ in the natural basis:

$$
\begin{aligned}
O & =\sum_{a+b=c+d} c_{a b, c d} \sum_{j}|a, b\rangle\left\langle c,\left.d\right|_{j, j+1}\right. \\
& =\sum_{a+b=c+d} c_{a b, c d} O_{a b, c d} .
\end{aligned}
$$

We can express $O_{a b, c d}$ as an MPO:

$$
\begin{aligned}
& O_{a b, c d}=\left(b_{l}^{O}\right)^{T}\left(\prod_{j=1}^{L} W_{a b, c d, j}\right) b_{r}^{O}, \\
& b_{l}^{O}=\left(\begin{array}{llllll}
1 & 0 & 0 & 1 & 0 & 0
\end{array}\right)^{T}, \\
& b_{r}^{O}=\left(\begin{array}{llllll}
0 & 0 & 1 & 0 & 0 & 1
\end{array}\right)^{T} . \\
& O_{a b, c d}|\mathcal{S}(z)\rangle=\sum_{\left\{\sigma_{j}\right\}}\left(\tilde{b}_{l}\right)^{T}\left(\prod_{j=1}^{L} B_{a b, c d}^{\left[\sigma_{j}\right]}\right) \tilde{b}_{r}\left|\left\{\sigma_{j}\right\}\right\rangle, \\
& B_{a b, c d}^{[\sigma]}=\sum_{\sigma^{\prime}} W_{a b, c d}^{\sigma, \sigma^{\prime}} \otimes A^{\left[\sigma^{\prime}\right]}, \tilde{b}_{l / r}=b_{l / r}^{O} \otimes b_{l / r} .
\end{aligned}
$$

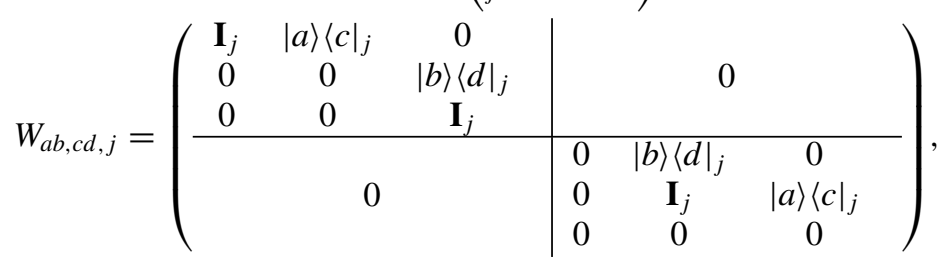

Then for $O=\sum_{a b, c d} c_{a b, c d} O_{a b, c d}$ we evaluate $|\psi\rangle=O|\mathcal{S}(z)\rangle$. We want $|\psi\rangle=0$, so it suffices to evaluate

$$
\langle\psi\rangle=\sum_{\begin{array}{c}
a+b=c+d, \\
a^{\prime}+b^{\prime}=c^{\prime}+d^{\prime}
\end{array}} c_{a^{\prime} b^{\prime}, c^{\prime} d^{\prime}}^{*} c_{a b, c d}\left\langle\mathcal{S}(z)\left|O_{a^{\prime} b^{\prime}, c^{\prime} d^{\prime}}^{\dagger} O_{a b, c d}\right| \mathcal{S}(z)\right\rangle .
$$

This becomes a linear problem in $c_{a b, c d}$, with matrix coefficients $\left\langle\mathcal{S}(z)\left|O_{a^{\prime} b^{\prime}, c^{\prime} d^{\prime}}^{\dagger} O_{a b, c d}\right| \mathcal{S}(z)\right\rangle$. We obtain this by forming the transfer matrix

$$
E_{a^{\prime} b^{\prime}, c^{\prime} d^{\prime} ; a b, c d}=\sum_{\sigma} B_{a^{\prime} b^{\prime}, c^{\prime} d^{\prime}}^{[\sigma] *} \otimes B_{a b, c d}^{[\sigma]}
$$

and calculating

$$
\begin{aligned}
\left\langle\mathcal{S}(z)\left|O_{a^{\prime} b^{\prime}, c^{\prime} d^{\prime}}^{\dagger} O_{a b, c d}\right| \mathcal{S}(z)\right\rangle & =\left(b_{l}^{E}\right)^{T} E_{a^{\prime} b^{\prime}, c^{\prime} d^{\prime} ; a b, c d}^{L} b_{r}^{E}, \\
b_{l / r}^{E} & =\tilde{b}_{l / r}^{*} \otimes \tilde{b}_{l / r} .
\end{aligned}
$$

The desired coefficients $c_{a b, c d}$ are the null eigenvectors of the Hermitian matrix $\left\langle\mathcal{S}(z)\left|O_{a^{\prime} b^{\prime}, c^{\prime} d^{\prime}}^{\dagger} O_{a b, c d}\right| \mathcal{S}(z)\right\rangle$. In practice it suffices to use the case $L=16$, because our operators are strictly nearest neighbor. (We have numerically verified that choosing larger $L$ does not give additional terms).
Given null eigenvectors $c_{a b, c d}$, we construct Hermitian operators by imposing the additional condition $c_{a b, c d}^{*}=c_{c d, a b}$. This procedure found the new operators 9-12 in Table II. Although this procedure was done for translationally invariant operators, only for no. 12 is translational invariance needed, while, similar to group A, terms 9-11 can be applied on each bond independently.

\section{Null operators}

One must take some care to recognize "null operators" in this nearest-neighbor operator basis, that is, operators that appear nontrivial but vanish under summation over $j$. For operators that conserve $S_{\text {Tot }}^{z}$, there are two linearly independent null operators:

$$
\begin{aligned}
& \sum_{j}(|1,-1\rangle\langle 1,-1|+| 1,0\rangle\langle 1,0| \\
& \quad-|-1,1\rangle\langle-1,1|-| 0,1\rangle\langle 0,1|)_{j, j+1} \\
& =\sum_{j}\left(|1\rangle\left\langle\left. 1\right|_{j}-\mid 1\right\rangle\left\langle\left. 1\right|_{j+1}\right)=0,\right. \\
& \sum_{j}(|0,-1\rangle\langle 0,-1|+| 0,1\rangle\langle 0,1| \\
& \quad-|-1,0\rangle\langle-1,0|-| 1,0\rangle\langle 1,0|)_{j, j+1} \\
& =\sum_{j}\left(|0\rangle\left\langle\left. 0\right|_{j}-\mid 0\right\rangle\left\langle\left. 0\right|_{j+1}\right)=0 .\right.
\end{aligned}
$$

The null operator corresponding to $\sum_{j}\left(|-1\rangle\left\langle-\left.1\right|_{j}-\right.\right.$ $|-1\rangle\left\langle-\left.1\right|_{j+1}\right)$ is linearly dependent in this basis on the above two terms. The MPS procedure finds all operators of the form Eq. (19) that annihilate $|\mathcal{S}(z)\rangle$ but by itself does not know that some of them are null operators, and as such these two terms will have to be subtracted from the null space obtained by this procedure.

For example, the procedure above gives the seemingly new term $\sum_{j}(|1,-1\rangle\langle 1,-1|-|-1,1\rangle\langle-1,1|)_{j, j+1}$ that 
annihilates $\left|\mathcal{S}_{N}\right\rangle$. This term also has an appealing interpretation of measuring the number of "left" domain walls " $1,-1$ " minus the number of "right" domain walls " $-1,1$," which always cancel in $\left|\mathcal{S}_{N}\right\rangle$ because there are no zeros. However, by Eq. (29), this term is actually equivalent to $\sum_{j}(|0,1\rangle\langle 0,1|-| 1,0\rangle\langle 1,0|)_{j, j+1}$, which is no. 10-no. 9 in Table II. We also note that of the four choices in nos. 9 and 10 only three are linearly independent, modulo Eq. (30).

For non- $S_{\text {Tot }}^{z}$-preserving operators, specifically those that change $S_{\text {Tot }}^{z}$ by 1 or 2 , the null operators are $\sum_{j} e^{i \phi}\left(|1\rangle\left\langle\left. 0\right|_{j}-\right.\right.$ $|1\rangle\left\langle\left. 0\right|_{j+1}\right)+$ H.c. and $\sum_{j} e^{i \phi}\left(|0\rangle\left\langle-\left.1\right|_{j}-\mid 0\right\rangle\left\langle-\left.1\right|_{j+1}\right)+\right.$ H.c., or $\sum_{j} e^{i \phi}\left(|1\rangle\left\langle-\left.1\right|_{j}-\mid 1\right\rangle\left\langle-\left.1\right|_{j+1}\right)+\right.$ H.c., respectively.

It is possible to eliminate the null operators from the start, e.g., by representing range- $k$ translationally invariant operators in the basis of length- $k$ "Gell-Mann strings" $\sum_{j} \lambda_{j}^{\left(\mu_{1}\right)} \cdots \lambda_{j+k-1}^{\left(\mu_{k}\right)}$ (spin-1 analogs of "Pauli strings" in spin$1 / 2$ chains), where the start of the string $\lambda^{\left(\mu_{1}\right)}$ must be a nontrivial Gell-Mann matrix while the other positions $\lambda^{\left(\mu_{i}\right)}, 2 \leqslant$ $i \leqslant k$ can also be an identity matrix. For range- 2 operators, our overcomplete basis choice with proper treatment of the null operators is equivalent but is more symmetric between the two sites and makes it somewhat easier to unpack results of the black-box search. A similar use of two-site operator basis (overcomplete for range- 2 translationally invariant operators) is also very convenient for the analytical argument in Appendix C.

\section{Discussion of results}

While it is easy to see that nos. 9-11 annihilate $\left|\mathcal{S}_{N}\right\rangle$ because $\left|\mathcal{S}_{N}\right\rangle$ contain no zeros, that no. 12 does is less immediate.

Number 12 can be viewed as an exchange of $1,-1$ and $-1,1$, with opposite sign for the two directions of the process. We can see that it annihilates $\left|\mathcal{S}_{N}\right\rangle$ by a preimage argument: The image only contains product states of $1 \mathrm{~s}$ and $-1 \mathrm{~s}$. There are equal numbers of $1,-1$ and $-1,1$ strings. For each “ $\pm 1, \mp 1$," there is a preimage where that string was “ $\mp 1, \pm 1$." Each of these preimages has the same sign in $\left|\mathcal{S}_{N}\right\rangle$, and therefore they cancel out in the image.

An alternate proof is the observation that

$$
\begin{aligned}
& i \text { no. } 12+\sum_{j}(|1,-1\rangle\langle 1,-1|-|-1,1\rangle\langle-1,1|)_{j, j+1} \\
& \quad=\sum_{j}(|1,-1\rangle-|-1,1\rangle)(\langle 1,-1|+\langle-1,1|)_{j, j+1}, \quad(31) \\
& \Rightarrow i \text { no. } 12=\sum_{j}((|1,-1\rangle-|-1,1\rangle)(\langle 1,-1|+\langle-1,1|) \\
& \quad+|1,0\rangle\langle 1,0|-| 0,1\rangle\langle 0,1|)_{j, j+1},
\end{aligned}
$$

with $\langle 1,-1|+\langle-1,1|,\langle 1,0|$, and $\langle 0,1|$ annihilating $\left|\mathcal{S}_{N}\right\rangle$. While no. 12 can be rewritten into a sum of local terms that annihilate $\left|\mathcal{S}_{N}\right\rangle$, this is only possible after the nonlocal rewriting in Eq. (29). This nonlocal cancellation is captured by our MPS method.

We remark that terms 1-7 and 9-11 correspond to the $S_{\text {Tot }}^{z}$ conserving part of the Shiraishi-Mori structure discussed in Refs. [31,34], while here we have additionally separated them into nonscarry [pseudospin-SU(2) symmetric] and true scarry terms. Nonlocal term 12 is new and does not appear to fit under this Shiraishi-Mori framework, at least with projectors in the space of nearest-neighbor operators.

We can show that operator 12 is indeed independent of this Shiraishi-Mori space as follows: Since no. 12 is purely imaginary, it suffices to show that it is independent of the other purely imaginary terms. These are nos. 5, 6, and 11 , with $\phi=\pi / 2$. Consider the state $\left|1,1, \ldots, 1,-1_{j}, 1, \ldots, 1\right\rangle$. While nos. 5 and 11 annihilate it and no. 6 can send it to either $i\left|1,1, \ldots, 0,0_{j}, 1, \ldots, 1\right\rangle \quad$ or $i\left|1,1, \ldots, 0_{j}, 0,1, \ldots, 1\right\rangle$, no. 12 maps this to $i\left|1,1, \ldots, 1,-1_{j-1}, 1, \ldots, 1\right\rangle-$ $i\left|1,1, \ldots, 1,-1_{j+1}, 1, \ldots, 1\right\rangle$. Thus, no. 12 indeed cannot be written as a superposition of nos. 5, 6, and 11 .

While terms 1-7 and 9-11 generalize to any bipartite graph, with arbitrary bond-dependent couplings, term 12 appears special to the $1 \mathrm{D}$ chain with $\mathrm{PBC}$ and with one coupling. However, from the nature of the scar states, we see that such a term placed on any closed loop of any bipartite lattice also annihilates the scar states, which allows us to generate analogs of no. 12 on any such lattice with arbitrary loop-dependent couplings. Equivalently, the term

$$
i \sum_{\langle\mathbf{i j}\rangle, \mathbf{i} \in A, \mathbf{j} \in B} f_{\mathbf{i j}}(|-1,1\rangle\langle 1,-1|-| 1,-1\rangle\langle-1,1|)_{\mathbf{i}, \mathbf{j}},
$$

with $\sum_{\mathbf{i}} f_{\mathbf{i j}}=0$ for all $\mathbf{i}$ from sublattice $A$ and $\sum_{\mathbf{j}} f_{\mathbf{i j}}=0$ for all $\mathbf{j}$ from sublattice $B$, annihilates the scar states. As an example, if every site on the bipartite lattice has even degree [79], we can achieve the above with $f_{\mathrm{ij}}= \pm 1$ by assigning an orientation to every bond such that on every site the number of inward bonds equals the number of outward bonds (a so-called Eulerian orientation).

As discussed in Appendix E, to find non- $S_{\mathrm{Tot}}^{z}$-preserving terms that annihilate $\left|\mathcal{S}_{N}\right\rangle$, it suffices to consider only terms that change $S_{\text {Tot }}^{z}$ by a fixed amount. We find that the following terms annihilate $\left|\mathcal{S}_{N}\right\rangle$ :

$$
\begin{aligned}
& (\alpha|0, \pm 1\rangle+\beta| \pm 1,0\rangle)\left\langle 0,\left.0\right|_{j, j+1}+\right.\text { H.c. } \\
& (\alpha|0, \pm 1\rangle+\beta| \pm 1,0\rangle)(\langle 1,-1|+\langle-1,1|)_{j, j+1}+\text { H.c., } \\
& (\alpha|0,1\rangle+\beta|1,0\rangle)(\gamma\langle 0,-1|+\delta\langle-1,0|)_{j, j+1}+\text { H.c. }
\end{aligned}
$$

for arbitrary complex $\alpha, \beta, \gamma, \delta$, after removing the null operators. These terms correspond to the non- $S_{\mathrm{Tot}}^{z}$-preserving part of the Shiraishi-Mori structure in Refs. [31,34], which hence exhausts such nearest-neighbor scarry Hamiltonians.

In Appendix $\mathrm{C}$ we provide an analytic derivation of the results in this section. We use this derivation in Appendix D to study the $k=0$ bimagnon tower: $\left|\mathcal{S}_{N}^{k=0}\right\rangle=\left(Q_{k=0}^{\dagger}\right)^{N}|\Omega\rangle$, where $Q_{k=0}^{\dagger}=\frac{1}{2} \sum_{j}\left(S_{j}^{+}\right)^{2}$. We further consider the related spin- $1 / 2 k=0$ magnon tower and find natural scarred models for both towers of states.

In Appendix E we prove that our systematic search is exhaustive in the space of nearest-neighbor models. In Appendix F we then systematically construct all spin-1 nearest-neighbor operators that annihilate the AKLT scar tower [28], revealing that the only family of nearest-neighbor models containing the 
AKLT scar tower is

$$
H_{\mathrm{AKLT}}+h S_{\mathrm{Tot}}^{z}+\sum_{j} \sum_{m, n=-2}^{0} c_{m, n}(j)\left|T_{2, m}\right\rangle\left\langle\left. T_{2, n}\right|_{j, j+1},\right.
$$

where $\left|T_{2,0}\right\rangle=(|1,-1\rangle+2|0,0\rangle+|-1,1\rangle) / \sqrt{6},\left|T_{2,-1}\right\rangle=$ $(|0,-1\rangle+|-1,0\rangle) / \sqrt{2}, \quad$ and $\quad\left|T_{2,-2}\right\rangle=|-1,-1\rangle, \quad$ and $c_{n m}(j)=c_{m n}^{*}(j)$ for Hermiticity but can vary with $j$. This corroborates the results in Refs. [30,34].

\section{ELECTRONIC MODELS WITH $\eta$-PAIRING SCAR TOWERS FROM SPIN-1 MODELS WITH XY TOWERS}

As discussed in Sec. III, the similarity between the electronic $\eta$-pairing operator $\eta^{\dagger}$ and the spin-1 operator $Q^{\dagger}$ suggests making the identification $|-1\rangle \rightarrow|h\rangle,|1\rangle \rightarrow|d\rangle$. We can identify $|0\rangle$ either with $|\uparrow\rangle$ or $|\downarrow\rangle$. This identification is useful to convert our results in Sec. IV into the electronic setting. By making the natural choice to restrict to electronic models with spin-SU(2)-invariant operators (which combined with electron number conservation imply separate conservation of both $\uparrow$ and $\downarrow$ species), we find that almost every spin- 1 model operator has a unique mapping to an electronic model operator.

\section{A. Ket-bra notation in electronic models}

Before we discuss the procedure we make a comment on notation. While in the spin setting the notation $|a, b\rangle\langle c, d|$ is unambiguous, in the electronic setting some care has to be taken. The notation $|a, b\rangle\langle c, d|$ is well defined when we restrict our discussion to nearest-neighbor terms in one dimension (and naturally to terms that conserve fermion number parity).

We adopt the Fock space ordering convention that the $c^{\dagger} \mathrm{s}$ should be ordered first with larger site numbers to the right, then with $\downarrow s$ to the right of $\uparrow s$. Therefore we identify the kets:

$$
\left.\left|\left\{n_{j, \alpha}\right\}\right\rangle=\left(c_{1, \uparrow}^{\dagger}\right)^{n_{1, \uparrow}}\left(c_{1, \downarrow}^{\dagger}\right)^{n_{1, \downarrow}}\left(c_{2, \uparrow}^{\dagger}\right)^{n_{2, \uparrow}}\left(c_{2, \downarrow}^{\dagger}\right)^{n_{2, \downarrow}} \ldots \mid \text { vac. }\right\rangle \text {. }
$$

We adopt the notation that (for the part of the ket associated with site $j)\left|h_{j}\right\rangle=\left|n_{j, \uparrow}=0, n_{j, \downarrow}=0\right\rangle,\left|d_{j}\right\rangle=$ $\left|n_{j, \uparrow}=1, n_{j, \downarrow}=1\right\rangle,\left|\uparrow_{j}\right\rangle=\left|n_{j, \uparrow}=1, n_{j, \downarrow}=0\right\rangle$, and $\left|\downarrow_{j}\right\rangle=$ $\left|n_{j, \uparrow}=0, n_{j, \downarrow}=1\right\rangle$.

Diagonal single-site operators are independent of the choice of convention:

$$
\begin{aligned}
|h\rangle\left\langle\left. h\right|_{j} \leftrightarrow\left(1-n_{j, \uparrow}\right)\left(1-n_{j, \downarrow}\right),\right. \\
|d\rangle\left\langle\left. d\right|_{j} \leftrightarrow n_{j, \uparrow} n_{j, \downarrow},\right. \\
|\uparrow\rangle\left\langle\left.\uparrow\right|_{j} \leftrightarrow n_{j, \uparrow}\left(1-n_{j, \downarrow}\right),\right.
\end{aligned}
$$

and likewise for $|\downarrow\rangle\left\langle\left.\downarrow\right|_{j}\right.$. However, our convention allows the ket-bra notation to be sensible for single-site off-diagonal terms and nearest-neighbor terms with even fermion parity. For example,

$$
\begin{array}{r}
|d\rangle\left\langle\left. h\right|_{j} \leftrightarrow c_{j \uparrow}^{\dagger} c_{j \downarrow}^{\dagger},\right. \\
|\uparrow\rangle\left\langle\left.\downarrow\right|_{j} \leftrightarrow c_{j, \uparrow}^{\dagger} c_{j, \downarrow},\right.
\end{array}
$$

etc. In particular, we have a direct connection between the $Q^{\dagger}$ and $\eta^{\dagger}$ operators, including all signs. However, terms like $|\uparrow\rangle\left\langle\left. h\right|_{j}\right.$ are not well defined (i.e., cannot appear in the electronic Hamiltonian by themselves), because they change total fermion parity. Hence it is natural to only consider operators where this does not happen.

We next consider two-site operators. We restrict ourselves to nearest-neighbor operators, because it is clear that, in general, operators $f_{j} g_{k}$ composed of fermionic operators $f_{j}$ and $g_{k}$ depend on the occupations between sites $j$ and $k$ and do not have well-defined (localized) ket-bra representations. Our Fock space convention allows unambiguous conversion between operator and ket-bra notation for nearest-neighbor terms. For example, consider the operator $c_{j, \uparrow}^{\dagger} c_{j+1, \uparrow} n_{j, \downarrow} n_{j+1, \downarrow}$, which sends $\left|\ldots \downarrow_{j}, d_{j+1} \ldots\right\rangle \rightarrow \pm\left|\ldots d_{j}, \downarrow_{j+1} \ldots\right\rangle$. The corresponding sign of $|d, \downarrow\rangle\left\langle\downarrow,\left.d\right|_{j, j+1}\right.$ is determined by writing

$$
\begin{aligned}
& c_{j, \uparrow}^{\dagger} c_{j+1, \uparrow} n_{j, \downarrow} n_{j+1, \downarrow}|\downarrow, d\rangle_{j, j+1} \\
& \left.=c_{j, \uparrow}^{\dagger} c_{j+1, \uparrow}\left(c_{j, \downarrow}^{\dagger} c_{j+1, \uparrow}^{\dagger} c_{j+1, \downarrow}^{\dagger} \mid \text { vac. }\right\rangle\right) \\
& \left.=-c_{j, \uparrow}^{\dagger} c_{j, \downarrow}^{\dagger} c_{j+1, \downarrow}^{\dagger} \mid \text { vac. }\right\rangle=-|d, \downarrow\rangle_{j, j+1} .
\end{aligned}
$$

Therefore $c_{j, \uparrow}^{\dagger} c_{j+1, \uparrow} n_{j, \downarrow} n_{j+1, \downarrow} \leftrightarrow-|d, \downarrow\rangle\left\langle\downarrow,\left.d\right|_{j, j+1}\right.$ [80].

The ket-bra notation is not well defined (does not give nice localized expressions) in every Fock space convention. Consider the convention where all up spins are listed before down spins, and are thus site ordered within each spin grouping. This convention, while convenient in numerical computations, is not a local one and thus does not support the nice ket-bra notation.

\section{B. Mapping spin-1 model operators to electronic model operators}

Having clarified the meaning of $|a, b\rangle\langle c, d|$ for fermions, we can map spin-1 model operators to electronic model operators. In each case we map $1 \rightarrow d,-1 \rightarrow h$, but the zero term requires some thought. There are several cases.

(1) Only $1 \mathrm{~s}$ and $-1 \mathrm{~s}$ appear in the ket and bra. Here the mapping is straightforward, and $d$ behaves as a boson hopping over $h$. Numbers 2, 7, and 12 fall in this case.

(2) One zero appears in the ket and bra each. To conserve each fermion spin species, we map the zero in both the bra and ket to the same species. Numbers 3-5 and 9-11 fall in this case, and we map, for example, $|0,1\rangle\langle 0,1|\rightarrow| \uparrow, d\rangle\langle\uparrow, d|$ or $|\downarrow, d\rangle\langle\downarrow, d|$.

(3) Two zeros appear in either the ket or the bra, e.g., $|00\rangle\langle 1,-1|$. To conserve the fermion numbers, $|00\rangle\langle 1,-1| \rightarrow$ $|\uparrow, \downarrow\rangle\langle d, h|$ or $|\downarrow, \uparrow\rangle\langle d, h|$. Number 6 belongs to this case.

(4) $|00\rangle\langle 00|$. Any " 00 " mappings that conserve the fermion numbers are allowed, e.g., $|\uparrow \uparrow\rangle\langle\uparrow \uparrow|,| \uparrow \downarrow\rangle\langle\downarrow, \uparrow|$, etc.; no. 1 belongs to this case.

Therefore we see that by just requiring separate conservation of $\uparrow$ and $\downarrow$ electron species each term has at most two possible mappings (except the $|00\rangle\langle 00| 00$ term). Further requiring spin-SU(2) invariance of the electronic terms leads to a unique choice of mapping, e.g., $|0,1\rangle\langle 1,0| \rightarrow$ $|\uparrow, d\rangle\langle d, \uparrow|+| \downarrow, d\rangle\langle d, \downarrow|$ and $(|1,-1\rangle+|-1,1\rangle)\langle 0,0| \rightarrow$ $(|d, h\rangle+|h, d\rangle)(\langle\uparrow, \downarrow|-\langle\downarrow, \uparrow|)$. One can see the required 
TABLE III. Translation of electronic operators from ket-bra notation to operator notation. Here we use the projector $P_{j, \sigma}$ as shorthand for $1-n_{j, \sigma}$.

\begin{tabular}{|c|c|c|}
\hline \multicolumn{3}{|c|}{ A. Operators that commute with $\eta^{\dagger}$} \\
\hline 1 & $\left|\sigma_{1}, \sigma_{2}\right\rangle\left\langle\sigma_{3},\left.\sigma_{4}\right|_{j, j+1}+\right.$ H.c., $\sigma_{1}+\sigma_{2}=\sigma_{3}+\sigma_{4}$ & $\mathbf{S}_{j} \cdot \mathbf{S}_{j+1}$ \\
\hline & & or $\left(n_{j, \uparrow}+n_{j, \downarrow}-2 n_{j, \uparrow} n_{j, \downarrow}\right)\left(n_{j+1, \uparrow}+n_{j+1, \downarrow}-2 n_{j+1, \uparrow} n_{j+1, \downarrow}\right)$ \\
\hline 2 & $(|d\rangle\langle d|+| h\rangle\langle h|)_{j}(|d\rangle\langle d|+| h\rangle\langle h|)_{j+1}$ & $4\left(\left(n_{j, \uparrow}-\frac{1}{2}\right)\left(n_{j, \downarrow}-\frac{1}{2}\right)+\frac{1}{4}\right)\left(\left(n_{j+1, \uparrow}-\frac{1}{2}\right)\left(n_{j+1, \downarrow}-\frac{1}{2}\right)+\frac{1}{4}\right)$ \\
\hline 3 & $(|d\rangle\langle d|+| h\rangle\langle h|)_{j}(|\uparrow\rangle\langle\uparrow|+| \downarrow\rangle\langle\downarrow|)_{j+1}$ & $2\left(\left(n_{j, \uparrow}-\frac{1}{2}\right)\left(n_{j, \downarrow}-\frac{1}{2}\right)+\frac{1}{4}\right)\left(n_{j+1, \uparrow} P_{j+1, \downarrow}+P_{j+1, \uparrow} n_{j+1, \downarrow}\right)$ \\
\hline 5 & $\begin{array}{l}e^{i \phi}(|\uparrow, d\rangle\langle d, \uparrow|+| \downarrow, d\rangle\langle d, \downarrow|-| \uparrow, h\rangle\langle h, \uparrow| \\
-|\downarrow, h\rangle\langle h, \downarrow|)_{j, j+1}+\text { H.c. }\end{array}$ & $-\sum_{\sigma} e^{i \phi}\left(c_{j+1, \sigma}^{\dagger} c_{j, \sigma} n_{j,-\sigma} n_{j+1,-\sigma}+c_{j, \sigma}^{\dagger} c_{j+1, \sigma} P_{j,-\sigma} P_{j+1,-\sigma}\right)+$ H.c. \\
\hline 6 & $e^{i \phi}(|\uparrow, \downarrow\rangle-|\downarrow, \uparrow\rangle)(\langle d, h|+\langle h, d|)_{j, j+1}+$ H.c. & $\sum_{\sigma} e^{i \phi}\left(c_{j+1, \sigma}^{\dagger} c_{j, \sigma} n_{j,-\sigma} P_{j+1,-\sigma}+c_{j, \sigma}^{\dagger} c_{j+1, \sigma} n_{j+1,-\sigma} P_{j,-\sigma}\right)+$ H.c. \\
\hline 7 & $(|d, h\rangle+|h, d\rangle)(\langle d, h|+\langle h, d|)_{j, j+1}$ & $n_{j, \uparrow} n_{j, \downarrow} P_{j+1, \uparrow} P_{j+1, \downarrow}+P_{j, \uparrow} P_{j, \downarrow} n_{j+1, \uparrow} n_{j+1, \downarrow}+\left(c_{j, \uparrow}^{\dagger} c_{j, \downarrow}^{\dagger} c_{j+1, \downarrow} c_{j+1, \uparrow}+\right.$ H.c. $)$ \\
\hline 9 & $|d\rangle\left\langle\left. d\right|_{j}(|\uparrow\rangle\langle\uparrow|+| \downarrow\rangle\langle\downarrow|)_{j+1}\left(\right.\right.$ or $|h\rangle\left\langle\left. h\right|_{j}\right)$ & $n_{j, \uparrow} n_{j, \downarrow}\left(n_{j+1, \uparrow} P_{j+1, \downarrow}+P_{j+1, \uparrow} n_{j+1, \downarrow}\right)\left(\right.$ or $P_{j, \uparrow} P_{j, \downarrow}$ instead of $\left.n_{j, \uparrow} n_{j, \downarrow}\right)$ \\
\hline 10 & $(|\uparrow\rangle\langle\uparrow|+| \downarrow\rangle\langle\downarrow|)_{j}|d\rangle\left\langle\left. d\right|_{j+1}\left(\right.\right.$ or $|h\rangle\left\langle\left. h\right|_{j+1}\right)$ & $\left(n_{j, \uparrow} P_{j, \downarrow}+P_{j, \uparrow} n_{j, \downarrow}\right) n_{j+1, \uparrow} n_{j+1, \downarrow}\left(\right.$ or $P_{j+1, \uparrow} P_{j+1, \downarrow}$ instead of $\left.n_{j+1, \uparrow} n_{j+1, \downarrow}\right)$ \\
\hline 11 & $e^{i \phi}(|\uparrow, d\rangle\langle d, \uparrow|+| \downarrow, d\rangle\langle d, \downarrow|)_{j, j+1}+$ H.c. & $-\sum_{\sigma} e^{i \phi} c_{j+1, \sigma}^{\dagger} c_{j, \sigma} n_{j,-\sigma} n_{j+1,-\sigma}+$ H.c. \\
\hline & (or $h$ instead of $d$ ) & (or $\sum_{\sigma} e^{i \phi} c_{j, \sigma}^{\dagger} c_{j+1, \sigma} P_{j,-\sigma} P_{j+1,-\sigma}+$ H.c.) \\
\hline 12 & $i \sum_{j}(|h, d\rangle\langle d, h|-| d, h\rangle\langle h, d|)_{j, j+1}$ & $\sum_{j}\left(i c_{j+1, \uparrow}^{\dagger} c_{j+1, \downarrow}^{\dagger} c_{j, \downarrow} c_{j, \uparrow}+\right.$ H.c. $)$ \\
\hline
\end{tabular}

signs most easily in the ket-bra notation, because we do not have to worry about signs when applying the electronic $S_{j}^{ \pm}$ operators onto kets [see Eq. (37)]. For example, for no. 6,

$$
\begin{aligned}
& {\left[S_{\mathrm{Tot}}^{+}, e^{i \phi}(|\uparrow, \downarrow\rangle+\gamma|\downarrow, \uparrow\rangle)(\langle d, h|+\langle h, d|)+\text { H.c. }\right]} \\
& =e^{i \phi}(1+\gamma)|\uparrow, \uparrow\rangle(\langle d, h|+\langle h, d|) \\
& \quad-e^{-i \phi}\left(1+\gamma^{*}\right)(|d, h\rangle+|h, d\rangle)\langle\downarrow, \downarrow| .
\end{aligned}
$$

This commutator is only zero when $\gamma=-1$, fixing the sign of the spin-SU(2)-invariant mapping.

The case of $|00\rangle\langle 00|$ is an exception. In this case there are two spin-SU(2)-symmetric terms allowed: $\mathbf{S}_{j} \cdot \mathbf{S}_{j+1}$ and $\sum_{\sigma, \sigma^{\prime}}\left|\sigma, \sigma^{\prime}\right\rangle\left\langle\sigma,\left.\sigma^{\prime}\right|_{j, j+1}=\left(n_{j, \uparrow}+n_{j, \downarrow}-2 n_{j, \uparrow} n_{j, \downarrow}\right)\left(n_{j+1, \uparrow}+\right.\right.$ $n_{j+1, \downarrow}-2 n_{j+1, \uparrow} n_{j+1, \downarrow}$ ) (the projector onto the space of no holons or doublons).

This allows us to complete the right column in Table II. By applying the procedure discussed above, we translate these operators in terms of standard electronic operators in Table III. A subtle point is that terms 5, 6, and 11 corresponding to single-electron hopping cannot be immediately mapped in their translationally invariant (i.e., summed over $j$ ) form, because there are sign ambiguities with hopping between sites 1 and $L$. However, because we can specialize to bondwise operators satisfying the desired commutation or scar annihilation properties, the mapping can proceed, by suitable redefining of the site numbers, or ordering convention, for hopping across the ends (for more details, see Sec. V C on generalizing these results to arbitrary bipartite lattices). Only no. 12 needs to be mapped in its translationally invariant form. Here the conversion is valid in PBC because the term moves two electrons and the simplistic ket-bra notation still holds across the end points.

While we verified directly in the fermion picture that the electronic terms have the desired properties with $\eta^{\dagger}$ or $\left|\psi_{N}\right\rangle$, these properties are in fact immediate from the spin-1 to electronic model translation. When considering group A, $Q^{\dagger}$ only involves $| \pm 1\rangle$ while $\eta^{\dagger}$ only involves $|d\rangle,|h\rangle$. Except for the case of $|00\rangle\langle 00|$, due to the electronic spin-SU(2) constraint, operators involving the "irrelevant" state $|0\rangle$ are mapped uniquely onto electronic operators involving $|\uparrow\rangle,|\downarrow\rangle$. Therefore we can establish a bijection between spin-1 model and electronic model nearest-neighbor operators, and the commutation result carries from one setting to the other.

Likewise, for group C, terms $9-11$ have $\uparrow / \downarrow$ in the bras of the ket-bra and trivially annihilate the $\eta$-pairing scar tower. Number 12 involves only $h$ and $d$, and the results from the spin-1 setting carry over with no issue.

A point of curiosity is that the analog of the minus sign in the spin- 1 model $H_{0}$ [Eq. (14), no. 5 with $\phi=0$ ] is naturally present in the electronic hopping terms with real-valued hopping amplitude. The relative minus sign is important for $H_{0}$ to be pseudospin $\mathrm{SU}(2)$ symmetric [and for the electronic model that it maps to to be $\eta$-pairing $\mathrm{SU}(2)$ symmetric]. In the spin-1 setting for physical spins it is less natural to have a sign difference between $|0,-1\rangle\langle-1,0|$ and $|0,1\rangle\langle 1,0|$ spin-exchange processes, and so the "natural" spin-1 XY model studied by Schecter and Iadecola breaks the pseudospin symmetry.

In electronic models, however, due to fermionic anticommutation, the electronic hopping Hamiltonian (no. 5) gives 
opposite signs to the terms $|\sigma, h\rangle\langle h, \sigma|$ and $|\sigma, d\rangle\langle d, \sigma|$. We recover the pure hopping term from no. 6-no. 5 in Table III (with $\phi=0$ in both terms), in agreement with the fact that the electronic hopping, and thus the Hubbard model, is $\eta$-pairing SU(2) symmetric.

To address our original goal of finding simplest models that turn the $\eta$-pairing states into true scar states, we additionally require that the operators respect inversion and time-reversal symmetry. This leaves us with the combinations of nos. 9 and $10-\sum_{j}(|\uparrow\rangle\langle\uparrow|+| \downarrow\rangle\langle\downarrow|)_{j}|d\rangle\left\langle\left. d\right|_{j+1}+\mid d\right\rangle\left\langle\left. d\right|_{j}(|\uparrow\rangle\langle\uparrow|+\right.$ $|\downarrow\rangle\langle\downarrow|)_{j+1}$ and its $h$ equivalent-and no. 11. One would expect the first term to come from a Coulomb interaction $\frac{1}{2} \sum_{j}\left(n_{j, \uparrow}+n_{j, \downarrow}\right)\left(n_{j+1, \uparrow}+n_{j+1, \downarrow}\right)$. The other contributions to the density-density term are $\frac{1}{2} \sum_{j, \sigma, \sigma^{\prime}}\left|\sigma, \sigma^{\prime}\right\rangle\left\langle\sigma,\left.\sigma^{\prime}\right|_{j, j+1}\right.$ and $2 \sum_{j}|d, d\rangle\left\langle d,\left.d\right|_{j, j+1}\right.$. The former is a possible translation of no. 1, but the latter is not in Table III. Therefore the Coulomb interaction does not preserve the $\eta$-pairing states as scars and we do not expect the first term to arise "naturally."

Term 11, however, is contained in the Hirsch term [Eq. (9)]. Specifically, from Table III, $H_{\text {Hirsch }}=X$ (no. 6-2 no. 11), with $\phi=0$. We therefore conclude that not only is the Hirsch model scarred but it is one of few models to have the $\eta$-pairing states as scars. The Hirsch model is also arguably the most "natural" model in this family of scarred models, having been previously explored for different reasons.

We lastly note that by adding no. 1 the $\eta$-pairing scars are robust to the presence of spin-spin interactions.

The procedure outlined in this paper gives a framework for listing families of models which share an exact scar tower. An interesting question for future study might be to see if one can then similarly systematically identify sets of states as candidate scar towers, on which we can apply this method.

\section{Generalization to an arbitrary bipartite lattice}

While the procedure in Sec. IV assumed translational invariance, the individual terms in nos. 1-7 and 9-11 across each bond $j, j+1$ satisfy the desired commutation or scar tower annihilation properties. In the spin-1 model setting, we can generalize these terms to any bond $\langle\mathbf{i j}\rangle$ between sites $\mathbf{i}$ and j on different bipartite components. This also turns out to be true in the electronic setting. There is no Fock space ordering convention that simultaneously preserves locality (in the sense discussed in this section) for every bond $\langle\mathbf{i}, \mathbf{j}\rangle$. However, for a given bond $\langle\mathbf{i j}\rangle$, we can define a convention that orders site $\mathbf{j}$ operators immediately after site $\mathbf{i}$ operators, for example. We can then define the ket-bra $|\ldots\rangle\left\langle\left.\ldots\right|_{\mathbf{i}, \mathbf{j}}\right.$ in this convention and prove the commutation or scar annihilation properties for the operator on this bond.

Lastly, as discussed in Sec. IVC2, we can generalize no. 12 onto bipartite lattices by writing it as an operator on closed loops in the graph.

\section{CONCLUSION}

We observed that in the Hirsch model the $\eta$-pairing states-exactly known states in the Hubbard model-become many-body scar states, because the $\eta$-pairing SU(2) symmetry is broken. While this observation is easy to verify, we arrived at this conclusion by first studying nearest-neighbor spin-1 models that are scarred by the spin-1 XY scar tower. Our systematic study separated models in which the spin-1 XY scar tower states are true scars from models that preserve the pseudospin SU(2) symmetry. We also found a new model (no. 12) that lies outside the established Shiraishi-Mori projector structure in the spin-1 XY model. These results give insights on other spin models with scar towers (Appendix D).

We then translated our findings from the spin-1 setting to the electronic setting, and obtained a family of models that are scarred by the $\eta$-pairing states, of which the Hirsch model is a member. This paper thus furthers our understanding of exact many-body scar towers and provides a systematic way of constructing families of scarred models.

Note added. Recently, we learned about related work by Moudgalya, Regnault, and Bernevig [81].

\section{ACKNOWLEDGMENTS}

We thank B. Andrei Bernevig, Anushya Chandran, Matthew Fisher, Jim Garrison, Vedika Khemani, Ryan Mishmash, Sanjay Moudgalya, and Nicolas Regnault for valuable discussions, and in particular Cheng-Ju Lin for many discussions and collaborations on related topics. We thank Hosho Katsura for valuable discussions and comments on the paper. D.K.M. acknowledges funding from the James C. Whitney SURF Fellowship, Caltech Student-Faculty Programs. This work was also supported by National Science Foundation Grant No. DMR-1619696.

\section{APPENDIX A: ADDITIONAL SCAR TOWERS IN THE HIRSCH MODEL}

In addition to the $\eta$-pairing states $\left|\psi_{N}\right\rangle$ in Eq. (2), we also find several more towers of scar states in the 1D Hirsch model [Eq. (9)]. We define these as

$$
\begin{gathered}
\left.\left|\psi_{N, M}\right\rangle=\left(t^{\dagger}\right)^{M}\left|\psi_{N}\right\rangle=\left(t^{\dagger}\right)^{M}\left(\eta^{\dagger}\right)^{N} \mid \text { vac. }\right\rangle, \\
\left.\left|\phi_{N}\right\rangle=s^{\dagger}\left|\psi_{N}\right\rangle=s^{\dagger}\left(\eta^{\dagger}\right)^{N} \mid \text { vac. }\right\rangle
\end{gathered}
$$

where

$$
\begin{aligned}
t^{\dagger} & =\sum_{j}(-1)^{j} c_{j, \uparrow}^{\dagger} c_{j+1, \uparrow}^{\dagger}, \\
s^{\dagger} & =\sum_{j}(-1)^{j}\left(c_{j, \uparrow}^{\dagger} c_{j+1, \downarrow}^{\dagger}-c_{j, \downarrow}^{\dagger} c_{j+1, \uparrow}^{\dagger}\right) .
\end{aligned}
$$

$t^{\dagger}$ creates a nearest-neighbor triplet $|\uparrow, \uparrow\rangle$ with momentum $\pi$, while $s^{\dagger}$ creates a nearest-neighbor singlet $|\uparrow, \downarrow\rangle-|\downarrow, \uparrow\rangle$ with momentum $\pi$. While $t^{\dagger}$ can be applied an arbitrary number of times to the $\eta$-pairing states $\left|\psi_{N}\right\rangle=\left|\psi_{N, 0}\right\rangle, s^{\dagger}$ can only be applied once to obtain the exact eigenstate $\left|\phi_{N}\right\rangle=$ $s^{\dagger}\left|\psi_{N}\right\rangle$.

$\left|\psi_{N, M}\right\rangle$ and $\left|\phi_{N}\right\rangle$ both have energies $E=N U$, independent of the strength of the Hirsch term $X$. (Here and below, we set $\mu=0$ without loss of generality.) Additionally, $\psi_{N, M}$ has total spin $S=M$, momentum $k=(N+M) \pi(\bmod 2 \pi)$, and site inversion number $I_{S}=1$, while $\phi_{N}$ has spin $S=0$, momentum $k=(N+1) \pi(\bmod 2 \pi)$, and site inversion number $I_{s}=-1$.

We prove that $\left|\psi_{N, M}\right\rangle$ and $\left|\phi_{N}\right\rangle$ are exact eigenstates using a commutator argument [34]. We first note that the states of interest all have exactly $N$ doublons. Hence, it suffices to 
show that they are eigenstates of - in fact annihilated bythe kinetic terms. In fact, since these states are independent of $X$, we show that each hopping process $H_{\mathrm{kin}, a}, a=1,2,3$ independently annihilates the scar towers, where

$$
\begin{aligned}
& H_{\text {kin, } 1}=\sum_{j, \sigma}\left(|h, \sigma\rangle\left\langle\sigma,\left.h\right|_{j, j+1}+\text { H.c. }\right),\right. \\
& H_{\text {kin }, 2}=\sum_{j}\left[(|d, h\rangle+|h, d\rangle)(\langle\uparrow, \downarrow|-\langle\downarrow, \uparrow|)_{j, j+1}+\text { H.c. }\right], \\
& H_{\text {kin }, 3}=\sum_{j, \sigma}\left(|d, \sigma\rangle\left\langle\sigma,\left.d\right|_{j, j+1}+\text { H.c. }\right) .\right.
\end{aligned}
$$

The expressions in terms of electron operators are obtained using the prescription described in Sec. V and Table III $\left(H_{\text {kin }, 1 / 3} \leftrightarrow\right.$ no. 11 and $H_{\text {kin, } 2} \leftrightarrow$ no. 6$)$. We take these electronic expressions with periodic boundary conditions, $c_{L+1, \sigma} \equiv c_{1, \sigma}$, as defining our model, but we use the above ket-bra writings in terms of $h, \uparrow, \downarrow, d$ as being more compact and better revealing structure in the arguments below. There is a small subtlety regarding hopping across the bond $(L, 1)$ [80]. Since our states $\left|\psi_{N, M}\right\rangle$ and $\left|\phi_{N}\right\rangle$ always have an even number of electrons, the hopping terms across $(L, 1)$ for $H_{\text {kin }, a}$ flip sign. For example, the $H_{\text {kin, } 1}$ term across $(L, 1)$ is $-\left(|h, \sigma\rangle\langle h, \sigma| \sigma, h_{L, 1}+\right.$ H.c. $)$. While in the rest of our proofs we do not explicitly exhibit this subtlety, it is easy to verify that appropriate ket-bra expressions for the operators $t^{\dagger}$ and $s^{\dagger}$ also have this flipped sign when the $\sigma, \sigma^{\prime}$ pair is created across the bond $(L, 1)$. These sign differences cancel out and our proofs remain valid.

The Hirsch Hamiltonian in Eq. (9) can be written as $H=$ $-t H_{\mathrm{kin}, 1}-(t-X) H_{\mathrm{kin}, 2}+(t-2 X) H_{\mathrm{kin}, 3}+U \sum_{j}|d\rangle\left\langle\left. d\right|_{j}\right.$. Given the fact that $\left|\psi_{0, M}\right\rangle$ and $\left|\phi_{0}\right\rangle$ are annihilated by $H_{\text {kin }, a}$ (which we will prove below), if we show that $\left[H_{\text {kin }, a}, \eta^{\dagger}\right]\left|\psi_{N, M}\right\rangle=\left[H_{\text {kin }, a}, \eta^{\dagger}\right]\left|\phi_{N}\right\rangle=0$, it follows that these states are annihilated by $H_{\mathrm{kin}, a}$.

From Table III we know that $\left[H_{\text {kin, }, 2}, \eta^{\dagger}\right]=0$ and $\left[H_{\text {kin, } 1}-H_{\text {kin, } 3}, \eta^{\dagger}\right]=0$. Then it suffices to show that $\left[H_{\text {kin }, 1}, \eta^{\dagger}\right]\left|\psi_{N, M}\right\rangle=\left[H_{\text {kin }, 1}, \eta^{\dagger}\right]\left|\phi_{N}\right\rangle=0$.
Evaluating the commutator gives

$$
\begin{aligned}
{\left[H_{\mathrm{kin}, 1}, \eta^{\dagger}\right]=} & \sum_{j, \sigma}\left[|h, \sigma\rangle\left\langle\sigma,\left.h\right|_{j, j+1}+\text { H.c., } \eta^{\dagger}\right]\right. \\
= & \sum_{j, \sigma}(-1)^{j}\left[| h , \sigma \rangle \langle \sigma , h | _ { j , j + 1 } + \text { H.c. } , | d \rangle \left\langle\left.h\right|_{j}\right.\right. \\
& -|d\rangle\left\langle\left. h\right|_{j+1}\right] \\
= & \sum_{j, \sigma}(-1)^{j}(|\sigma, d\rangle\langle h, \sigma| \\
& -|d, \sigma\rangle\langle\sigma, h|)_{j, j+1} .
\end{aligned}
$$

This annihilates $\left|\psi_{N, M}\right\rangle$ and $\left|\phi_{N}\right\rangle$ because we note that in these states the spins appear in bound pairs, as elaborated below.

In $\left|\psi_{N, M}\right\rangle$, there are only $\uparrow$ unpaired electrons, which appear in nearest-neighbor pairs $\uparrow, \uparrow$. Then

$$
\begin{aligned}
& \sum_{\sigma}(|\sigma, d\rangle\langle h, \sigma|-| d, \sigma\rangle\langle\sigma, h|)_{j, j+1}\left|\psi_{N, M}\right\rangle \\
& =\left(|\uparrow, d, \uparrow\rangle\left\langle h, \uparrow,\left.\uparrow\right|_{j, j+1, j+2}-\mid \uparrow, d, \uparrow\right\rangle\langle\right. \\
& \left.\quad \uparrow, \uparrow,\left.h\right|_{j-1, j, j+1}\right)\left|\psi_{N, M}\right\rangle .
\end{aligned}
$$

Summing over $j$, we get for $\left[H_{\mathrm{kin}, 1}, \eta^{\dagger}\right]\left|\psi_{N, M}\right\rangle$

$$
\begin{aligned}
& -\sum_{j}(-1)^{j}|\uparrow, d, \uparrow\rangle\left(\left\langle h, \uparrow, \uparrow|+\langle\uparrow, \uparrow, h|)_{j-1, j, j+1} \mid \psi_{N, M}\right\rangle\right. \\
& =0 .
\end{aligned}
$$

The last line follows because the $\uparrow, \uparrow$ pair created by $t^{\dagger}$ has momentum $k=\pi$, and so such pairs appear in $\left|\psi_{N, M}\right\rangle$ in the superposition $|h, \uparrow, \uparrow\rangle-|\uparrow, \uparrow, h\rangle$.

In $\left|\phi_{n}\right\rangle$, there is one pair of nearest-neighbor $\uparrow$ and $\downarrow$ electrons in a singlet state. Then

$$
\begin{aligned}
& \sum_{\sigma}(|\sigma, d\rangle\langle h, \sigma|-| d, \sigma\rangle\langle\sigma, h|)_{j, j+1}\left|\phi_{N}\right\rangle \\
&= {\left[(|\uparrow, d, \downarrow\rangle\langle h, \uparrow, \downarrow|+| \downarrow, d, \uparrow\rangle\langle h, \downarrow, \uparrow|)_{j, j+1, j+2}\right.} \\
&\left.-(|\downarrow, d, \uparrow\rangle\langle\downarrow, \uparrow, h|+| \uparrow, d, \downarrow\rangle\langle\uparrow, \downarrow, h|)_{j-1, j, j+1}\right]\left|\phi_{N}\right\rangle .
\end{aligned}
$$

Summing over $j$ gives for $\left[H_{\mathrm{kin}, 1}, \eta^{\dagger}\right]\left|\phi_{N}\right\rangle$

$$
\begin{aligned}
& -\sum_{j}(-1)^{j}\left[|\uparrow, d, \downarrow\rangle(\langle h, \uparrow, \downarrow|+\langle\uparrow, \downarrow, h|)_{j-1, j, j+1}\right. \\
& \left.\quad+|\downarrow, d, \uparrow\rangle(\langle h, \downarrow, \uparrow|+\langle\downarrow, \uparrow, h|)_{j-1, j, j+1}\right]\left|\phi_{N}\right\rangle=0 .
\end{aligned}
$$

This is zero because the $\uparrow, \downarrow$ and $\downarrow, \uparrow$ pairs have momentum $\pi$.

We lastly have to show that the initial states $\left|\psi_{0, M}\right\rangle$ and $\left|\phi_{0}\right\rangle$ are annihilated by each $H_{\text {kin, } a}$. The case of $\left|\psi_{0, M}\right\rangle=$ $\left(t^{\dagger}\right)^{M} \mid$ vac. $\rangle$ is immediate. The only nontrivial term is $H_{\text {kin, } 1}$. Since the bound $\uparrow, \uparrow$ pairs in $\left|\psi_{0, M}\right\rangle$ can be constructed by creating electrons in momentum states of the form $c_{\uparrow, k}^{\dagger} c_{\uparrow, \pi-k}^{\dagger}$, the kinetic energy of which is $\cos (k)+\cos (\pi-k) \stackrel{1}{=} 0$, we conclude that $\left|\psi_{0, M}\right\rangle$ has zero energy under $H_{\text {kin,1 }}$. 
In the case of $\left|\phi_{0}\right\rangle=s^{\dagger} \mid$ vac. $\rangle$, we have to consider both $H_{\text {kin, } 1}$ and $H_{\text {kin, } 2}$. To show $H_{\text {kin, } 1}\left|\phi_{0}\right\rangle=0$, we write

$$
\begin{gathered}
H_{\text {kin }, 1} \sum_{j}(-1)^{j}\left(\left|\ldots, h, \uparrow_{j}, \downarrow, h, \ldots\right\rangle-\left|\ldots, h, \downarrow_{j}, \uparrow, h, \ldots\right\rangle\right) \\
=\sum_{j}(-1)^{j}\left[\left|\ldots, \uparrow, h_{j}, \downarrow, h, \ldots\right\rangle-\left|\ldots, \downarrow, h_{j}, \uparrow, h, \ldots\right\rangle\right. \\
\left.\quad+\left|\ldots, h, \uparrow_{j}, h, \downarrow, \ldots\right\rangle-\left|\ldots, h, \downarrow_{j}, h, \uparrow, \ldots\right\rangle\right]=0 .
\end{gathered}
$$

Lastly, $H_{\text {kin, } 2}\left|\phi_{0}\right\rangle=0$ because

$$
\begin{aligned}
& H_{\text {kin }, 2} \sum_{j}(-1)^{j}\left(\left|\ldots, h, \uparrow_{j}, \downarrow, h, \ldots\right\rangle-\left|\ldots, h, \downarrow_{j}, \uparrow, h, \ldots\right\rangle\right) \\
& =2 \sum_{j}(-1)^{j}\left(\left|\ldots, h, d_{j}, h, h, \ldots\right\rangle+\left|\ldots, h, h_{j}, d, h, \ldots\right\rangle\right) \\
& =0 .
\end{aligned}
$$

Note that the arguments for $\left[H_{\mathrm{kin}, 1}, \eta^{\dagger}\right]\left|\phi_{N}\right\rangle=0$ and $H_{\text {kin, },}\left|\phi_{0}\right\rangle=0$ held for the $|\uparrow, \downarrow\rangle$ and $|\downarrow, \uparrow\rangle$ pairs separately; we did not require them to be in a singlet. Their triplet combination is a spin rotation of $\left|\psi_{0,1}\right\rangle=t^{\dagger} \mid$ vac. $\rangle$ and is also annihilated by $H_{\text {kin }, a}$.

We remark that we numerically observe other entanglement entropy outlier states. Specifically, we observe states with the same energy and in the same symmetry sector as $\left|\phi_{N}\right\rangle$ which appear to contain $N$ doublons and an $X$ dependent superposition of a long-range entangled singlet. There are also entanglement entropy outliers at $k \neq 0, \pi$ and $S=0$. However, these states are not states of well-defined doublon number and do not have simple energies. A detailed study of these outlier states could be interesting future work.

Lastly, we note that terms 6-8 and 5 and 11 (with $\phi=0$ ) in Table III preserve both scar towers. Additionally, the towers $\left|\phi_{N}\right\rangle$ and $\left|\psi_{N, 1}\right\rangle$ are preserved by nos. 2-4, and both SU(2)invariant choices of no. 1 . Unlike the $\eta$-pairing states, since these new states involve pairs across bonds, hopping terms "perpendicular" to these pairs do not cancel, and so we do not expect them to generalize to higher dimensions.

\section{Analogous new scar tower in the spin-1 XY model}

Given the similarities identified between the spin- $1 \mathrm{XY}$ and Hubbard models, it might not be surprising that in the $1 \mathrm{D}$ spin-1 XY model there is a tower of states analogous to the "singlet" tower $\left|\phi_{N}\right\rangle$. Specifically, defining a state

$$
\left|\mathcal{S}^{00}\right\rangle=\sum_{j}(-1)^{j}\left|-1, \ldots,-1,0_{j}, 0,-1, \ldots,-1\right\rangle,
$$

the states

$$
\left|\mathcal{S}_{N}^{00}\right\rangle=\left(Q^{\dagger}\right)^{N}\left|\mathcal{S}^{00}\right\rangle
$$

are also zero-energy eigenstates of $H_{\mathrm{XY}}$. Similar to the singlet and "triplet" scar towers in the Hirsch model, this can be proven using the commutator argument and commutation relation [34]

$$
\begin{aligned}
{\left[H_{\mathrm{XY}}, Q^{\dagger}\right]=} & J \sum_{j}(-1)^{j}(|0,1\rangle\langle-1,0|-| 1,0\rangle \\
& \times\langle 0,-1|)_{j, j+1} .
\end{aligned}
$$

Since the zero only occurs in a single 00 in all of these states, we can write

$$
\begin{aligned}
& |0,1\rangle\left\langle-1,\left.0\right|_{j, j+1} \mid \mathcal{S}_{N}^{00}\right\rangle \\
& \quad=|0,1,0\rangle\left\langle-1,0,\left.0\right|_{j, j+1, j+2} \mid \mathcal{S}_{N}^{00}\right\rangle, \\
& |1,0\rangle\left\langle 0,-\left.1\right|_{j, j+1} \mid \mathcal{S}_{N}^{00}\right\rangle \\
& \quad=|0,1,0\rangle\left\langle 0,0,-\left.1\right|_{j-1, j, j+1} \mid \mathcal{S}_{N}^{00}\right\rangle .
\end{aligned}
$$

This gives

$$
\begin{aligned}
{\left[H_{\mathrm{XY}}, Q^{\dagger}\right]\left|\mathcal{S}_{N}^{00}\right\rangle=} & J \sum_{j}(-1)^{j}|0,1,0\rangle(\langle-1,0,0| \\
& +\langle 0,0,-1|)_{j, j+1, j+2}\left|\mathcal{S}_{N}^{00}\right\rangle .
\end{aligned}
$$

The last line is zero because the 00 in $\left|\mathcal{S}_{N}^{00}\right\rangle$ has momentum $\pi$. The commutator argument is complete by noting that $H_{\mathrm{XY}}$ annihilates the base of the tower $\left|\mathcal{S}^{00}\right\rangle$, again because the 00 has momentum $\pi$. This tower is similar to the singlet tower in that only one 00 can be present in the tower of states. Accordingly, there is no analog to the triplet towers of states $\left|\psi_{N, M}\right\rangle$ in the spin-1 XY model. We lastly note that due to special symmetries present in the $1 \mathrm{D}$ spin-1 XY model [31,32] we have to add a longer-range term such as $\sum_{j}(|0,0,1\rangle+|1,0,0\rangle)(\langle 0,0,1|+\langle 1,0,0|)_{j-1, j, j+1}$ for this tower of states to be "true scars," that is, states in a quantum chaotic spectrum.

\section{APPENDIX B: SYSTEMATIC CONSTRUCTION OF SPIN MODELS THAT COMMUTE WITH $Q^{\dagger}$}

In this Appendix we obtain the set of nearest-neighbor operators that commute with $Q^{\dagger}$ (Sec. IV A). Restricting to Hermitian operators $O,\left[O, Q^{\dagger}\right]=0$ implies that $\left[O, S_{\mathrm{Tot}}^{z}\right]=0$. We express the operators $O=\sum_{j} o_{j}$ in terms of the two-site bases $|a, b\rangle\langle a, b| c, d_{j, j+1}, a+b=c+d$. It suffices to consider the commutator $\left[o_{j},\left[\left(S_{j}^{+}\right)^{2}-\left(S_{j+1}^{+}\right)^{2}\right] / 2\right]=\left[o_{j}, q_{j}^{\dagger}\right] \cdot q_{j}^{\dagger}$ connects sectors $S_{j}^{z}+S_{j+1}^{z}=-2,0,2$ and $-1,1$, so we can consider these groups separately.

The $S_{j}^{z}+S_{j+1}^{z}=-2,0,2$ group gives

$$
\begin{aligned}
& \left.o_{j}=\left(\begin{array}{ccccc}
a & & & & \\
& b & c & d & \\
& c^{*} & e & f & |1,1\rangle \\
& d^{*} & f^{*} & g & \\
& & & & h
\end{array}\right)_{j, j+1}|1,-1\rangle, 0\right\rangle, \\
& q_{j}^{\dagger}=\left(\begin{array}{rrrrr}
0 & -1 & 0 & 1 & 0 \\
& & & & 1 \\
& & & & 0 \\
& & & & -1
\end{array}\right)_{j, j+1} .
\end{aligned}
$$

The matrix basis is indicated in Eq. (B1)-for example, the " $d$ " entry indicates the term $d|1,-1\rangle\left\langle-1,\left.1\right|_{j, j+1}\right.$. 
Evaluating the commutator, we get

$$
\left[o_{j}, q_{j}^{\dagger}\right]=\left(\begin{array}{ccccc}
0 & b-d^{*}-a & c-f^{*} & a+d-g & 0 \\
& & & b-d-h \\
& & & c^{*}-f \\
& & & d^{*}+h-g \\
& & & 0
\end{array}\right)_{j, j+1} .
$$

For $\left[O, Q^{\dagger}\right]=0$, each matrix entry must be zero. We obtain the linearly independent operators 1, 2, 6, and 7 in Table II.

Performing a similar analysis for the $S_{j}^{z}+S_{j+1}^{z}=-1,1$ group gives terms 3, 4, and 5 in Table II.

\section{APPENDIX C: ANALYTIC PROOF OF SPIN-1 XY SCARRED MODELS}

In Sec. IV, we performed a brute force numerical search to find the family of all translationally invariant nearest-neighbor models that contain the spin-1 XY scar tower (Table II). While the numerical search can be generalized to other scar towers, such as the AKLT scar towers in Appendix F, in this Appendix we analytically prove our result in Sec. IV.

It will be convenient to use the following basis for spin-1, two-site states [34]:

$$
\begin{aligned}
& \left|X_{1}\right\rangle=(|1,-1\rangle+|-1,1\rangle) / \sqrt{2}, \quad\left|X_{2}\right\rangle=|0,0\rangle, \\
& \left|X_{3}\right\rangle=|1,0\rangle, \quad\left|X_{4}\right\rangle=|0,1\rangle, \\
& \left|X_{5}\right\rangle=|-1,0\rangle, \quad\left|X_{6}\right\rangle=|0,-1\rangle, \\
& \left|X_{7}\right\rangle=(|1,-1\rangle-|-1,1\rangle) / \sqrt{2}, \\
& \left|X_{8}\right\rangle=|1,1\rangle, \quad\left|X_{9}\right\rangle=|-1,-1\rangle .
\end{aligned}
$$

We are interested in finding operators that annihilate all $\left|\mathcal{S}_{N}\right\rangle$. We first note that $|\ldots\rangle\left\langle X_{i}\right|$ for $1 \leqslant i \leqslant 6$ trivially annihilates the scar tower $\left|\mathcal{S}_{N}\right\rangle$. So it suffices to consider the action of $|\ldots\rangle\left\langle X_{i}\right|, 7 \leqslant i \leqslant 9$ on $\left|\mathcal{S}_{N}\right\rangle$.

As in Table II, we restrict our attention to terms that preserve $S_{\text {Tot }}^{z}$. The terms $\sum_{j}\left|X_{8}\right\rangle\left\langle\left. X_{8}\right|_{j, j+1}\right.$ and $\left.\sum_{j} \mid X_{9}\right\rangle\left\langle\left. X_{9}\right|_{j, j+1}\right.$ do not annihilate the ferromagnetic states $\left|\mathcal{S}_{L}\right\rangle=|1,1, \ldots, 1\rangle$ and $|\Omega\rangle=|-1,-1, \ldots,-1\rangle$, respectively (and are the only such terms, so their action cannot be canceled out). Therefore we only need to consider terms in the linear space $\sum_{j}\left\{\left|X_{1}\right\rangle\left\langle X_{7}|,| X_{2}\right\rangle\left\langle X_{7}|,| X_{7}\right\rangle\left\langle X_{7}\right|\right\}_{j, j+1}$. Just by considering annihilation of the first state of the tower, $\left|\mathcal{S}_{1}\right\rangle=Q^{\dagger}|\Omega\rangle$, we conclude that the only Hermitian term we can construct from this space and its Hermitian conjugates is $\sum_{j} c\left|X_{1}\right\rangle\left\langle X_{1}\right| X_{7 j, j+1}+$ H.c.. The choices $c=1$ and $i$ span this space. The $c=1$ choice is in fact related to simpler terms $\sum_{j}\left(\left|X_{4}\right\rangle\left\langle X_{4}|-| X_{3}\right\rangle\left\langle X_{3}\right|\right)_{j, j+1}$ [see Eq. (29) in Sec. IVC1], while the $c=i$ choice is proportional to no. 12 in Table. II. That no. 12 annihilates all scar states $\left|\mathcal{S}_{N}\right\rangle$ was proven in Sec. IVC2.

This exhausts the space of nearest-neighbor, translationally invariant operators that annihilate $\left|\mathcal{S}_{N}\right\rangle$, and we recover the results in Table II.

\section{APPENDIX D: COMPLETE FAMILY OF SPIN-1 MODELS SCARRED WITH THE $\boldsymbol{k}=0$ BIMAGNON TOWER}

The analytical argument in Appendix $C$ can also be used to find all models that are scarred by the " $k=0$ bimagnon tower." By this we mean the tower of states

$$
\begin{aligned}
\left|\mathcal{S}_{N}^{k=0}\right\rangle & =\left(Q_{k=0}^{\dagger}\right)^{N}|\Omega\rangle, \\
Q_{k=0}^{\dagger} & =\frac{1}{2} \sum_{j}\left(S_{j}^{+}\right)^{2}, \quad|\Omega\rangle=|-1, \ldots,-1\rangle .
\end{aligned}
$$

The $Q_{k=0}^{\dagger}$ is similar to the $Q^{\dagger}$ operator in the main text $\left(Q^{\dagger} \equiv Q_{k=\pi}^{\dagger}\right)$, except that it imparts zero momentum, instead of momentum $\pi$. Given that two distinct models-the spin-1 XY and spin-1 AKLT models - contain towers related by $Q^{\dagger}$, it is natural to ask if there are any physically interesting models that host the $k=0$ bimagnon tower of states. We find that, without one special term, any nearest-neighbor model containing the $k=0$ bimagnon tower conserves the number of zeros $n_{0}$, and hence can be mapped, in the $n_{0}=0$ symmetry sector, to a family of spin- $1 / 2$ models with arbitrary Heisenberg interactions and special Dzyaloshinskii-Moriyatype interaction.

Repeating the argument in Appendix C, we can analytically find all nearest-neighbor models that annihilate $\left|\mathcal{S}_{N}^{k=0}\right\rangle$. Using the notation in Eq. (C1), we first note that any term $|\ldots\rangle\left\langle X_{i}\right|$ for $2 \leqslant i \leqslant 7$ annihilates $\left|\mathcal{S}_{N}^{k=0}\right\rangle$. For $S_{\text {Tot }}^{z}$-preserving Hamiltonians, besides the above trivially annihilating terms, we only need to consider terms $\sum_{j}\left|X_{8}\right\rangle\left\langle\left. X_{8}\right|_{j, j+1}, \sum_{j} \mid X_{9}\right\rangle\left\langle\left. X_{9}\right|_{j, j+1}\right.$ and in the linear space $\sum_{j}\left\{\left|X_{1}\right\rangle\left\langle X_{1}|,| X_{2}\right\rangle\left\langle X_{1}|,| X_{7}\right\rangle\left\langle X_{1}\right|\right\}_{j, j+1}$. As in the $k=\pi$ bimagnon case, we can prove that the only Hermitian terms we can construct using this set that annihilate the $k=0$ bimagnon tower are $\sum_{j} c\left|X_{1}\right\rangle\left\langle X_{7}\right|+$ H.c. Of these, only the $c=i$ choice gives a term independent of the previously considered terms $|\ldots\rangle\left\langle X_{i}\right|$ with $2 \leqslant i \leqslant 7$. Thus, the term

$$
\begin{aligned}
& i \sum_{j}\left(\left|X_{1}\right\rangle\left\langle X_{7}|-| X_{7}\right\rangle\left\langle X_{1}\right|\right)_{j, j+1} \\
& \quad=i \sum_{j}(|-1,1\rangle\langle 1,-1|-| 1,-1\rangle\langle-1,1|)_{j, j+1} \\
& \quad=\frac{i}{4} \sum_{j}\left[\left(S_{j}^{-}\right)^{2}\left(S_{j+1}^{+}\right)^{2}-\left(S_{j}^{+}\right)^{2}\left(S_{j+1}^{-}\right)^{2}\right]
\end{aligned}
$$

is the only new term that annihilates the $k=0$ bimagnon tower, and unlike the other terms the mechanism of annihilation is nonlocal. 
In this set of operators, there is only one term that does not conserve the number of zeros:

$$
c\left|X_{2}\right\rangle\left\langle X_{7}\right|+\text { H.c. }=c|0,0\rangle(\langle 1,-1|-\langle-1,1|) / \sqrt{2}+\text { H.c. }
$$

This term is antisymmetric under spatial inversion and in general more difficult to come by than the $k=\pi$ case. For the $k=\pi$ bimagnon tower, the corresponding term is $c|0,0\rangle(\langle 1,-1|+\langle-1,1|)+$ H.c., which is present in the $H_{\mathrm{XY}}$ spin-exchange term [see Eq. (15)].

However, Eq. (D6), with $c=i$, can arise as part of a natural-looking DMI:

$$
\begin{aligned}
& h_{j, j+1}^{\mathrm{DMI}}=\hat{\mathbf{z}} \cdot\left(\mathbf{S}_{j} \times \mathbf{S}_{j+1}\right)=S_{j}^{x} S_{j+1}^{y}-S_{j}^{y} S_{j+1}^{x} \\
& =i\left(\sqrt{2}\left|X_{7}\right\rangle\left\langle X_{2}|+| X_{3}\right\rangle\left\langle X_{4}|+| X_{6}\right\rangle\left\langle X_{5}\right|\right)_{j, j+1}+\text { H.c. },
\end{aligned}
$$

where the additional parts also annihilate the $k=0$ bimagnon tower. On the other hand, the case $c=1$ corresponds to a less natural-looking term:

$$
i\left(S_{j}^{x} S_{j+1}^{y}-S_{j}^{y} S_{j+1}^{x}\right) S_{j}^{z} S_{j+1}^{z}+\text { H.c. } \sim\left|X_{7}\right\rangle\left\langle X_{2}\right|+\text { H.c. }
$$

which in addition breaks physical time-reversal invariance and will not be considered further. On a 1D chain with only nearest-neighbor interactions, the former DMI model is in fact unitarily related to the XY chain of Schecter and Iadecola [31], by the transformation $H_{\mathrm{XY}}=U H_{\mathrm{DMI}} U^{-1}$ [82], with

$$
U=\prod_{j} \exp \left(i \frac{\pi}{2} j S_{j}^{z}\right)=\otimes_{j}\left(\begin{array}{ccc}
i^{j} & 0 & 0 \\
0 & 1 & 0 \\
0 & 0 & (-i)^{j}
\end{array}\right)_{j}
$$

in open boundary conditions and PBCs for $L=4 n$. The same unitary relates also the $k=0$ and $\pi$ bimagnon towers: $U\left|\mathcal{S}_{N}^{k=0}\right\rangle \propto\left|\mathcal{S}_{N}\right\rangle$. (For PBC in $L=4 n+2, H_{\text {DMI }}$ rotates to an $\mathrm{XY}$ chain with antiperiodic boundary conditions. Because the proof for these terms to annihilate the XY scar tower in Sec. IV relied on strictly local annihilation, the antiperiodic XY model, with flipped sign on $-S_{L}^{x} S_{1}^{x}-S_{L}^{y} S_{1}^{y}$, also contains the $k=\pi$ bimagnon tower.)

Furthermore, thinking directly about models with $k=0$ bimagnon towers, it is clear that the above term does not require bipartite structure and in general is not simply derived from the spin-1 XY model.

To summarize, analogously to how the model $H_{\mathrm{XY}}+$ $h S_{\mathrm{Tot}}^{z}+D \sum_{\mathbf{j}}\left(S_{\mathbf{j}}^{z}\right)^{2}$ [Eq. (12)] hosts the $k=\pi$ tower of scars in any dimension, we expect the model $H_{\mathrm{DMI}}+h S_{\mathrm{Tot}}^{z}+$ $D \sum_{\mathbf{j}}\left(S_{\mathbf{j}}^{z}\right)^{2}$ to host the $k=0$ tower of scars in any dimension. In one dimension, just as we need to introduce the additional term $H_{3}=J_{3} \sum_{j}\left(S_{j}^{x} S_{j+3}^{x}+S_{j}^{y} S_{j+3}^{y}\right)$ to break additional symmetries, we need to likewise introduce a range- $k$ DMI term $H_{k}^{\prime}=\sum_{j}\left(S_{j}^{x} S_{j+k}^{y}-S_{j}^{y} S_{j+k}^{x}\right)$ to break the equivalent symmetry (because we do not need bipartiteness, $k$ can be even or odd here).

Finally, the term in Eq. (D5) can also be generalized to higher dimensions, by placing it on oriented loops (maintaining the same coupling along the loop), exactly as in the generalization of the term 12 to higher dimensions in Sec. IVC2.

\section{Application: Generalization of models with $\pi$-bimagnon towers to arbitrary graphs}

In the main text, we considered models realizing $\pi$ bimagnon towers on bipartite lattices, with two-site terms defined only on links connecting different sublattices A and B. We can immediately generalize these to models with two-site terms defined also on links connecting sites on the same sublattice, A-A or B-B. Indeed, from the point of view of one such sublattice, the $\pi$-bimagnon states look like the $k=0$ bimagnon states considered in this Appendix. Hence, all terms considered above, placed on either A-A or B-B links, will preserve the $\pi$-bimagnon tower of Schecter and Iadecola.

\section{Application: Reduction to new spin-1/2 models with a magnon scar tower}

In the absence of the $c\left|X_{2}\right\rangle\left\langle X_{2}\right| X_{7}+$ H.c. term [Eq. (D6)], a model containing the $k=0$ bimagnon scar tower will preserve the number of zeros $n_{0}$. The scar tower will lie in the $n_{0}=0$ sector. In this sector, the only relevant terms are those that only involve $1 \mathrm{~s}$ and $-1 \mathrm{~s}$. These terms can be mapped onto a spin-1/2 model, replacing 1 with $\uparrow$ and -1 with $\downarrow$.

There are three terms in the identified family that contain only 1 s and -1 s. They are

$$
\begin{aligned}
& \left|X_{7}\right\rangle\left\langle\left. X_{7}\right|_{j, j+1} \mapsto(|\uparrow, \downarrow\rangle\langle\uparrow, \downarrow|+| \downarrow, \uparrow\rangle\langle\downarrow, \uparrow|\right. \\
& -|\uparrow, \downarrow\rangle\langle\downarrow, \uparrow|-| \downarrow, \uparrow\rangle\langle\uparrow, \downarrow|)_{j, j+1} \\
& =\frac{1}{2}\left(I_{j, j+1}-\mathbf{s}_{j} \cdot \mathbf{s}_{j+1}\right), \\
& \sum_{j}\left|X_{1}\right\rangle\left\langle\left. X_{7}\right|_{j, j+1}+\right.\text { H.c. } \\
& \mapsto \sum_{j}(|\uparrow \downarrow\rangle\langle\uparrow \downarrow|-| \downarrow \uparrow\rangle\langle\downarrow \uparrow|)_{j, j+1} \\
& =0 \text {, } \\
& i \sum_{j}\left|X_{1}\right\rangle\left\langle\left. X_{7}\right|_{j, j+1}+\text { H.c. } \mapsto i \sum_{j}\left(s_{j}^{-} s_{j+1}^{+}-s_{j}^{+} s_{j+1}^{-}\right)\right. \\
& =2 \sum_{j}\left(s_{j}^{y} s_{j+1}^{x}-s_{j}^{x} s_{j+1}^{y}\right),
\end{aligned}
$$

where we use $\mathbf{s}_{j}$ to denote spin-1/2 spin operators. Equation (D10) can be seen because $\sum_{j}\left|X_{1}\right\rangle\left\langle\left. X_{7}\right|_{j, j+1}+\right.$ H.c. maps to a counting of opposite domain walls $N_{\uparrow \downarrow}-N_{\downarrow \uparrow}=0$, which is zero in a closed spin- $1 / 2$ chain. The term in Eq. (D9) corresponds to a spin-1/2 Heisenberg model, while the term in Eq. (D11) corresponds to a DMI of spin-1/2s and also corresponds to term 12 in Table II.

Therefore, if the $c\left|X_{2}\right\rangle\left\langle X_{7}\right|+$ H.c. term is not present, a spin- 1 model scarred by the $k=0$ bimagnon tower is equivalent to, in the $n_{0}=0$ sector, a spin- $1 / 2$ Heisenberg model with Dzyaloshinskii-Moriya interaction. On the $1 \mathrm{D}$ chain with only nearest-neighbor terms, this model is integrable [83], by essentially undoing the spin "twist" in the DMI and transforming to an XXZ chain with twisted boundary conditions, which is in turn solvable by the Bethe ansatz.

The spin- 1 model $k=0$ bimagnon tower maps onto the simple spin-1/2 model $k=0$ magnon tower, generated by 
repeated action of the operator $\sum_{j} s_{j}^{+}$on the state $|\downarrow, \downarrow, \ldots, \downarrow\rangle$. This tower is nothing else but the familiar $S=\mathrm{Vol} / 2$ highest-spin multiplet which would describe the degenerate ferromagnetic states in an SU(2)-invariant model.

We conclude from these observations that any inversionsymmetric, nearest-neighbor spin-1 model cannot contain the spin-1 $k=0$ bimagnon tower as scars: it will possess $n_{0}$ conservation and be equivalent to the spin- $1 / 2$ Heisenberg model in the $n_{0}=0$ sector. The bimagnon states are not scars because of the spin- $1 / 2 \mathrm{SU}(2)$ symmetry in the $n_{0}=0$ sectors.

However, there are interesting spin-1/2 models scarred by the $k=0$ magnon tower. These states will be eigenstates of any spin-SU(2)-invariant model, for example, the $J_{1}-J_{2}$ model:

$$
H_{J_{1}-J_{2}}=\sum_{j}\left(J_{1} \mathbf{s}_{j} \cdot \mathbf{s}_{j+1}+J_{2} \mathbf{s}_{j} \cdot \mathbf{s}_{j+2}\right) .
$$

We can then add the spin- $1 / 2$ DM interaction to get a simple nonintegrable spin- $1 / 2$ model scarred by the $k=0$ magnon tower:

$$
H_{J 1-J 2}+D H_{\mathrm{DMI}}, \quad H_{\mathrm{DMI}}=\sum_{j} \hat{\mathbf{z}} \cdot\left(\mathbf{s}_{j} \times \mathbf{s}_{j+1}\right) .
$$

This model has been considered in the context of the magnetoelectric effect in ferroelectric materials [84-86] and is believed to describe materials such as $\mathrm{LiCuVO}_{4}[87,88]$ and $\mathrm{LiCu}_{2} \mathrm{O}_{2}[89,90]$. The DM interaction breaks the spin-SU(2) symmetry, and the presence of this multiplet is a nontrivial "scar" property. We lastly note that with fine-tuned nearestneighbor and next-nearest-neighbor DM terms Ref. [91] found an additional tower of exact eigenstates corresponding to "spiral" spin states, focusing on regimes when these are the ground states. Reference [91] also noticed our observation here that the DMI preserves the $k=0$ magnon tower.

We verified numerically that this model in one dimension is indeed scarred by the $k=0$ magnon scar tower. Apart from translational invariance and $S^{z}$ conservation, there is an additional spin-flip plus inversion symmetry given by $g=$ $\prod_{j} \sigma_{j}^{x} \times I$, where $I\left|s_{1}, s_{2}, \ldots, s_{L}\right\rangle=\left|s_{L}, s_{L-1}, \ldots, s_{1}\right\rangle$. However, for small $D$, the couplings $J_{1}$ and $J_{2}$ must have opposite signs in order for the ferromagnetic states to be in the bulk of the spectrum. In our numerical study with couplings $J_{1}=$ $1, J_{2}=-0.6, D=0.3$, this was indeed the case for the scar state in the sector $k=0, S_{\mathrm{Tot}}^{z}=0, g=1$. We note that the " $\mathrm{CuO}_{2}$ ribbon chain" materials [87-90,92] have the desired opposite signs of $J_{1}$ and $J_{2}$.

We note that the same scar states were also obtained in a Shiraishi-Mori type "toy model" in Ref. [18] with complicated four-spin interactions. We found a much simpler and more realistic model with only two-spin interactions; also, our model does not appear to be of Shiraishi-Mori type with two-site projectors.

This model can be extended to higher dimensions, as long as the DMI terms occur in loops. That is, orienting the DMI vectors with $+\hat{z}$, each directed bond $\mathbf{i} \rightarrow \mathbf{j}$ in the term $s_{\mathbf{i}}^{y} s_{\mathbf{j}}^{x}-$ $s_{\mathbf{i}}^{x} s_{\mathbf{j}}^{y}$ belongs to a unique closed loop $\mathbf{i} \rightarrow \mathbf{j} \rightarrow \mathbf{k} \cdots \rightarrow \mathbf{i}$. This is in fact the case for the DMI studied in the kagome lattice material herbertsmithite [93-95] and the triangular lattice material $\mathrm{Cs}_{2} \mathrm{CuCl}_{4}$ studied in Ref. [96], for example. Again, if all nearest-neighbor and further-neighbor Heisenberg couplings are antiferromagnetic, we expect that for small DMI these eigenstates are ceiling states and nominally not scars, but they are still special.

We also note that we can easily prepare a suitable initial state for perfect revivals in such scarred systems. Following Schecter and Iadecola's initial state for the spin-1 XY scars, we can give the ferromagnetic scars an equal energy splitting with the term $h S_{\text {Tot }}^{z}$. The ferromagnetic state in the $x$ direction $\otimes_{j}\left[(|\uparrow\rangle+|\downarrow\rangle)_{j} / \sqrt{2}\right]$ is a superposition of the $k=0$ magnon states and hence will experience perfect revivals.

Finally, we can also consider spin- $1 / 2$ models that contain the spin- $1 / 2 k=\pi$ magnon tower. The DMI term corresponds to no. 12 in Table II and also annihilates the $k=\pi$ magnon tower. Instead of the Heisenberg term, we we need to consider $\sum_{j}\left|X_{1}\right\rangle\left\langle\left. X_{1}\right|_{j, j+1}\right.$ instead, which corresponds to a less natural XXZ model $\sum_{j}\left(s_{j}^{x} s_{j+1}^{x}+s_{j}^{y} s_{j+1}^{y}-s_{j}^{z} s_{j+1}^{z}\right)$. Therefore, in the spin- $1 / 2$ context, it is more natural to consider models scarred by the $k=0$ magnon tower, instead of the $k=\pi$ one.

\section{APPENDIX E: EXHAUSTIVE SEARCH FOR MODELS SCARRED BY A GIVEN SCAR TOWER}

In this Appendix we provide a framework to exhaustively find all models that contain a given scar tower, provided the scar tower satisfies certain conditions. We then apply this method in Appendix F to find a complete family of nearestneighbor models scarred with the AKLT scars.

We are given a scar tower $\left\{\left|\mathcal{S}_{N}\right\rangle\right\}$, obtained by $\left|\mathcal{S}_{N}\right\rangle=$ $\left(Q^{\dagger}\right)^{N}\left|\mathcal{S}_{0}\right\rangle$, for some operator $Q^{\dagger}$ and state $\left|\mathcal{S}_{0}\right\rangle$. We then want to find a family of all models such that the $\left|\mathcal{S}_{N}\right\rangle$ are eigenstates with energy $E_{N}=q N+\lambda$, for some $q$ and $\lambda$. Without loss of generality, we can set $\lambda=0$ by subtracting $\lambda I$. We observe that for any such model $H$ we have that

$$
\forall N,\left[H, Q^{\dagger}\right]\left|\mathcal{S}_{N}\right\rangle=q Q^{\dagger}\left|\mathcal{S}_{N}\right\rangle, H\left|\mathcal{S}_{0}\right\rangle=0 .
$$

Suppose we have $H_{Q}$ such that

$$
\left[H_{Q}, Q^{\dagger}\right]=Q^{\dagger}, H_{Q}\left|\mathcal{S}_{0}\right\rangle=0 .
$$

We can then split $H$ into parts:

$$
H=q H_{Q}+H^{\prime}
$$

where

$$
\forall N,\left[H^{\prime}, Q^{\dagger}\right]\left|\mathcal{S}_{N}\right\rangle=0 .
$$

In our case of interest, $Q^{z}=S_{\mathrm{Tot}}^{z} / 2$ satisfies $\left[Q^{z}, Q^{\dagger}\right]=Q^{\dagger}$. Furthermore, in our examples, $\left|\mathcal{S}_{N}\right\rangle$ are eigenstates of $Q^{z}$.

It follows that $\left|\mathcal{S}_{0}\right\rangle$ is also an eigenstate of $H^{\prime}$ (the eigenvalue of which we can again set to zero). Then Eq. (E4) is equivalent to

$$
\forall N, H^{\prime}\left|\mathcal{S}_{N}\right\rangle=0 .
$$

It suffices to consider operators $H^{\prime}$ that change the magnetization $S_{\text {Tot }}^{z}$ by a fixed amount. This is because we require $H^{\prime}$ to annihilate each $\left|S_{N}\right\rangle$ individually. Since each $\left|S_{N}\right\rangle$ has fixed magnetization, if $H^{\prime}$ had components changing $S_{\text {Tot }}^{z}$ by a different amount, the images, upon action of different such components, would be in different $S_{\text {Tot }}^{z}$ sectors and would have 
to independently cancel, i.e., each component independently annihilates all $\left|S_{N}\right\rangle$.

Restricting to operators that change $S_{\text {Tot }}^{z}$ by a fixed amount has the following advantage: searching for all such $H^{\prime}$ is equivalent to finding all Hamiltonians that annihilate a compression of the tower states $\sum_{N} c_{N}\left|\mathcal{S}_{N}\right\rangle$. In the case of the AKLT model scar tower discussed below, this compression can be written as an MPS, allowing us to use the methods discussed in Sec. IV C. We lastly note that the identity should be trivially added to this list $H^{\prime}$, since it was used to set all eigenvalues to zero.

\section{APPENDIX F: COMPLETE FAMILY OF NEAREST-NEIGHBOR MODELS SCARRED WITH AKLT SCARS}

Our systematic search for models scarred with the spin-1 XY scar tower revealed the new term 12 that cannot be reduced to local terms annihilating these scar states. Motivated by this observation, in this Appendix we derive the complete family of nearest-neighbor models that contain the AKLT tower of scar states. These scar states are defined by acting the same operator $Q^{\dagger}$ in Eq. (13) on the AKLT ground state $|G\rangle$. It can be compactly expressed by the MPS [29]:

$$
|G\rangle=\sum_{\left\{\sigma_{1} \cdots \sigma_{L}\right\}} \operatorname{Tr}\left(A^{\left[\sigma_{1}\right]} \cdots A^{\left[\sigma_{L}\right]}\right)\left|\sigma_{1} \cdots \sigma_{L}\right\rangle,
$$

where

$$
\begin{aligned}
A^{[-1]} & =\sqrt{\frac{2}{3}}\left(\begin{array}{ll}
0 & 1 \\
0 & 0
\end{array}\right), \quad A^{[0]}=\frac{1}{\sqrt{3}}\left(\begin{array}{cc}
-1 & 0 \\
0 & 1
\end{array}\right) \\
A^{[1]} & =\sqrt{\frac{2}{3}}\left(\begin{array}{cc}
0 & 0 \\
-1 & 0
\end{array}\right) .
\end{aligned}
$$

The scar states in the AKLT model have energies $E_{n}=2 n$ and are given by [28]

$$
\left|\mathcal{S}_{n}\right\rangle=\left(Q^{\dagger}\right)^{n}|G\rangle
$$

For the AKLT model, it is convenient to work in the basis of states $\left\{\left|T_{J, M}\right\rangle\right\}$ of well-defined total spin $J$ and magnetization $M$ across two sites [34]:

$$
\begin{aligned}
\left|T_{2,-2}\right\rangle & =|-1,-1\rangle,\left|T_{2,-1}\right\rangle=\frac{1}{\sqrt{2}}(|0,-1\rangle+|-1,0\rangle), \\
\left|T_{2,0}\right\rangle & =\frac{1}{\sqrt{6}}(|1,-1\rangle+2|0,0\rangle+|-1,1\rangle), \\
\left|T_{2,1}\right\rangle & =\frac{1}{\sqrt{2}}(|1,0\rangle+|0,1\rangle),\left|T_{2,2}\right\rangle=|1,1\rangle, \\
\left|T_{1,-1}\right\rangle & =\frac{1}{\sqrt{2}}(|0,-1\rangle-|-1,0\rangle) \\
\left|T_{1,0}\right\rangle & =\frac{1}{\sqrt{2}}(|1,-1\rangle-|-1,1\rangle), \\
\left|T_{1,1}\right\rangle & =\frac{1}{\sqrt{2}}(|1,0\rangle-|0,1\rangle), \\
\left|T_{0,0}\right\rangle & =\frac{1}{\sqrt{3}}(|1,-1\rangle-|0,0\rangle+|-1,1\rangle) .
\end{aligned}
$$

As discussed in Appendix E, it suffices to find all terms that annihilate $\left|\mathcal{S}_{n}\right\rangle$. To do so we compress all $\left|\mathcal{S}_{n}\right\rangle$ into a single MPS with the MPO in Eq. (17), then use the MPS method outlined in Sec. IV C. By considering translationally invariant nearest-neighbor operators, as in the main text, we found that the following terms annihilate $\left|\mathcal{S}_{n}\right\rangle$ :

$$
\begin{gathered}
H_{0}=\sum_{j}\left[(|0,1\rangle\langle 1,0|-| 0,-1\rangle\langle-1,0|)_{j, j+1}+\text { H.c. }\right], \\
\left|T_{2,-2}\right\rangle\left\langle\left. T_{2,-2}\right|_{j, j+1}, \mid T_{2,-1}\right\rangle\left\langle\left. T_{2,-1}\right|_{j, j+1}, \mid T_{2,0}\right\rangle\left\langle\left. T_{2,0}\right|_{j, j+1},\right. \\
e^{i \phi}\left|T_{2,-2}\right\rangle\left\langle\left. T_{2,-1}\right|_{j, j+1}+\text { H.c., } e^{i \phi} \mid T_{2,-2}\right\rangle\left\langle\left. T_{2,0}\right|_{j, j+1}+\right.\text { H.c., } \\
e^{i \phi}\left|T_{2,-1}\right\rangle\left\langle\left. T_{2,0}\right|_{j, j+1}+\right.\text { H.c. }
\end{gathered}
$$

As in the spin-1 XY model case, most of these terms annihilate $\left|\mathcal{S}_{n}\right\rangle$ bondwise, as already written above. Unlike the spin-1 XY model case, however, the $H_{0}$ term needs its sum over $j$. In addition, $H_{0}$ and $\left|T_{2,0}\right\rangle\left\langle T_{2,0}\right| j, j+1$ commute with $Q^{\dagger}$. However, since the scar states here do not have a special relationship with the pseudospin symmetry (namely, they are not eigenstates of the total pseudospin), this property of the two terms is less important [34]. To get the scar energies $E_{n}=2 n$ we can add $S_{\text {Tot }}^{z}$. In fact, the AKLT model is contained in this space, by the fact that [34]

$$
\begin{aligned}
\frac{1}{2} H_{0}+S_{\mathrm{Tot}}^{z} & =\sum_{j}\left(\left|T_{2,2}\right\rangle\left\langle T_{2,2}|+| T_{2,1}\right\rangle\left\langle T_{2,1}|-| T_{2,-1}\right\rangle\left\langle T_{2,-1}|-| T_{2,-2}\right\rangle\left\langle T_{2,-2}\right|\right)_{j, j+1} \\
& =H_{\mathrm{AKLT}}-\sum_{j}\left(\left|T_{2,0}\right\rangle\left\langle T_{2,0}|+2| T_{2,-1}\right\rangle\left\langle T_{2,-1}|+2| T_{2,-2}\right\rangle\left\langle T_{2,-2}\right|\right)_{j, j+1} .
\end{aligned}
$$

We can then express the family of Hamiltonians scarred by the AKLT tower as

$$
H_{\mathrm{AKLT}}+h S_{\mathrm{Tot}}^{z}+\sum_{j} \sum_{m, n=-2}^{0} c_{m, n}(j)\left|T_{2, m}\right\rangle\left\langle\left. T_{2, n}\right|_{j, j+1},\right.
$$

reproducing the results from Refs. [30,34]. 
[1] J. M. Deutsch, Phys. Rev. A 43, 2046 (1991).

[2] M. Srednicki, Phys. Rev. E 50, 888 (1994).

[3] D. M. Basko, I. L. Aleiner, and B. L. Altshuler, Ann. Phys. (NY) 321, 1126 (2006).

[4] V. Oganesyan and D. A. Huse, Phys. Rev. B 75, 155111 (2007).

[5] J. H. Bardarson, F. Pollmann, and J. E. Moore, Phys. Rev. Lett. 109, 017202 (2012).

[6] M. Serbyn, Z. Papić, and D. A. Abanin, Phys. Rev. Lett. 110, 260601 (2013).

[7] M. Serbyn, Z. Papić, and D. A. Abanin, Phys. Rev. Lett. 111, 127201 (2013).

[8] D. A. Huse, R. Nandkishore, and V. Oganesyan, Phys. Rev. B 90, 174202 (2014).

[9] B. Bauer and C. Nayak, J. Stat. Mech. (2013) P09005.

[10] R. Nandkishore and D. A. Huse, Annu. Rev. Condens. Matter Phys. 6, 15 (2015).

[11] D. A. Abanin, E. Altman, I. Bloch, and M. Serbyn, Rev. Mod. Phys. 91, 021001 (2019).

[12] H. Bernien, S. Schwartz, A. Keesling, H. Levine, A. Omran, H. Pichler, S. Choi, A. S. Zibrov, M. Endres, M. Greiner, V. Vuletić, and M. D. Lukin, Nature (London) 551, 579 (2017).

[13] C. J. Turner, A. A. Michailidis, D. A. Abanin, M. Serbyn, and Z. Papić, Nat. Phys. 14, 745 (2018).

[14] C. J. Turner, A. A. Michailidis, D. A. Abanin, M. Serbyn, and Z. Papić, Phys. Rev. B 98, 155134 (2018).

[15] V. Khemani, C. R. Laumann, and A. Chandran, Phys. Rev. B 99, 161101(R) (2019).

[16] W. W. Ho, S. Choi, H. Pichler, and M. D. Lukin, Phys. Rev. Lett. 122, 040603 (2019).

[17] C.-J. Lin and O. I. Motrunich, Phys. Rev. Lett. 122, 173401 (2019).

[18] S. Choi, C. J. Turner, H. Pichler, W. W. Ho, A. A. Michailidis, Z. Papić, M. Serbyn, M. D. Lukin, and D. A. Abanin, Phys. Rev. Lett. 122, 220603 (2019).

[19] F. M. Surace, P. P. Mazza, G. Giudici, A. Lerose, A. Gambassi, and M. Dalmonte, Phys. Rev. X 10, 021041 (2020).

[20] T. Iadecola, M. Schecter, and S. Xu, Phys. Rev. B 100, 184312 (2019).

[21] N. Shiraishi, J. Stat. Mech. (2019) 083103.

[22] A. A. Michailidis, C. J. Turner, Z. Papić, D. A. Abanin, and M. Serbyn, Phys. Rev. X 10, 011055 (2020).

[23] S. Moudgalya, B. A. Bernevig, and N. Regnault, arXiv:1906.05292 (2019).

[24] C.-J. Lin, A. Chandran, and O. I. Motrunich, Phys. Rev. Res. 2, 033044 (2020).

[25] K. Bull, J.-Y. Desaules, and Z. Papić, Phys. Rev. B 101, 165139 (2020).

[26] A. A. Michailidis, C. J. Turner, Z. Papić, D. A. Abanin, and M. Serbyn, Phys. Rev. Res. 2, 022065 (2020).

[27] C.-J. Lin, V. Calvera, and T. H. Hsieh, Phys. Rev. B 101, 220304(R) (2020).

[28] S. Moudgalya, S. Rachel, B. A. Bernevig, and N. Regnault, Phys. Rev. B 98, 235155 (2018).

[29] S. Moudgalya, N. Regnault, and B. A. Bernevig, Phys. Rev. B 98, 235156 (2018).

[30] S. Moudgalya, E. O'Brien, B. A. Bernevig, P. Fendley, and N. Regnault, Phys. Rev. B 102, 085120 (2020).

[31] M. Schecter and T. Iadecola, Phys. Rev. Lett. 123, 147201 (2019).
[32] S. Chattopadhyay, H. Pichler, M. D. Lukin, and W. W. Ho, Phys. Rev. B 101, 174308 (2020).

[33] T. Iadecola and M. Schecter, Phys. Rev. B 101, 024306 (2020).

[34] D. K. Mark, C.-J. Lin, and O. I. Motrunich, Phys. Rev. B 101, 195131 (2020).

[35] N. Shiraishi and T. Mori, Phys. Rev. Lett. 119, 030601 (2017).

[36] T. Mori and N. Shiraishi, Phys. Rev. E 96, 022153 (2017).

[37] A. J. A. James, R. M. Konik, and N. J. Robinson, Phys. Rev. Lett. 122, 130603 (2019).

[38] V. Khemani, M. Hermele, and R. Nandkishore, Phys. Rev. B 101, 174204 (2020).

[39] A. Hudomal, I. Vasić, N. Regnault, and Z. Papić, Commun. Phys. 3, 99 (2020).

[40] N. Pancotti, G. Giudice, J. I. Cirac, J. P. Garrahan, and M. C. Bañuls, Phys. Rev. X 10, 021051 (2020).

[41] S. Pai and M. Pretko, Phys. Rev. Lett. 123, 136401 (2019).

[42] P. Sala, T. Rakovszky, R. Verresen, M. Knap, and F. Pollmann, Phys. Rev. X 10, 011047 (2020).

[43] N. Shibata, N. Yoshioka, and H. Katsura, Phys. Rev. Lett. 124, 180604 (2020).

[44] B. Mukherjee, S. Nandy, A. Sen, D. Sen, and K. Sengupta, Phys. Rev. B 101, 245107 (2020).

[45] A. Haldar, D. Sen, R. Moessner, and A. Das, arXiv:1909.04064 (2019).

[46] S. Sugiura, T. Kuwahara, and K. Saito, arXiv:1911.06092 (2019).

[47] H. Zhao, J. Vovrosh, F. Mintert, and J. Knolle, Phys. Rev. Lett. 124, 160604 (2020).

[48] K. Mizuta, K. Takasan, and N. Kawakami, arXiv:2004.04431 (2020).

[49] C. N. Yang, Phys. Rev. Lett. 63, 2144 (1989).

[50] O. Vafek, N. Regnault, and B. A. Bernevig, SciPost Phys. 3, 043 (2017).

[51] C. N. Yang and S. Zhang, Mod. Phys. Lett. B 4, 759 (1990).

[52] J. R. Garrison, R. V. Mishmash, and M. P. A. Fisher, Phys. Rev. B 95, 054204 (2017).

[53] T. Veness, F. H. L. Essler, and M. P. A. Fisher, Phys. Rev. B 96, 195153 (2017).

[54] T. Veness, F. H. L. Essler, and M. P. A. Fisher, Phil. Trans. R. Soc. A 375, 20160433 (2017).

[55] X. Yu, D. Luo, and B. K. Clark, Phys. Rev. B 98, 115106 (2018).

[56] T. Iadecola and M. Žnidarič, Phys. Rev. Lett. 123, 036403 (2019).

[57] F. H. L. Essler, H. Frahm, F. Göhmann, A. Klümper, and V. E. Korepin, The One-Dimensional Hubbard Model (Cambridge University, Cambridge, England, 2005).

[58] S.-Q. Shen and Z.-M. Qiu, Phys. Rev. Lett. 71, 4238 (1993).

[59] J. de Boer and A. Schadschneider, Phys. Rev. Lett. 75, 4298 (1995).

[60] S. Kitamura and H. Aoki, Phys. Rev. B 94, 174503 (2016).

[61] K. Li, arXiv:1901.06914 (2019).

[62] J. Li, D. Golez, P. Werner, and M. Eckstein, arXiv:1908.08693 (2019).

[63] T. Kaneko, T. Shirakawa, S. Sorella, and S. Yunoki, Phys. Rev. Lett. 122, 077002 (2019).

[64] J. E. Hirsch, Physica C 158, 326 (1989).

[65] J. E. Hirsch and F. Marsiglio, Phys. Rev. B 39, 11515 (1989).

[66] R. Micnas, J. Ranninger, and S. Robaszkiewicz, Phys. Rev. B 39, 11653 (1989). 
[67] R. Micnas, J. Ranninger, and S. Robaszkiewicz, Rev. Mod. Phys. 62, 113 (1990).

[68] R. Strack and D. Vollhardt, Phys. Rev. Lett. 70, 2637 (1993).

[69] J. de Boer, V. E. Korepin, and A. Schadschneider, Phys. Rev. Lett. 74, 789 (1995).

[70] B. R. Bułka, Phys. Rev. B 57, 10303 (1998).

[71] J. Vidal and B. Douçot, Phys. Rev. B 65, 045102 (2001).

[72] J. Hubbard, Proc. R. Soc. A 276, 238 (1963).

[73] L.-M. Duan, Europhys. Lett. 81, 20001 (2007).

[74] L. Arrachea and A. A. Aligia, Phys. Rev. Lett. 73, 2240 (1994).

[75] A. Schadschneider, Phys. Rev. B 51, 10386 (1995).

[76] Y. Y. Atas, E. Bogomolny, O. Giraud, and G. Roux, Phys. Rev. Lett. 110, 084101 (2013).

[77] A. Kitazawa, K. Hijii, and K. Nomura, J. Phys. A 36, L351 (2003).

[78] E. Chertkov and B. K. Clark, Phys. Rev. X 8, 031029 (2018).

[79] R. Diestel, in Graph Theory (Springer, New York, 2017), pp. 1-34.

[80] For an electronic chain with periodic boundary conditions, terms that contain single-electron hopping across the $(L, 1)$ bond acquire an additional sign $(-1)^{N_{\text {el.tot. }}-1}$ when going between the electron operator and ket-bra expressions compared to $(j, j+1)$ terms with $j<L$.

[81] S. Moudgalya, N. Regnault, and B. A. Bernevig, Phys. Rev. B 102, 085140 (2020).

[82] H. Karimi and I. Affleck, Phys. Rev. B 84, 174420 (2011).

[83] F. C. Alcaraz and W. F. Wreszinski, J. Stat. Phys. 58, 45 (1990).
[84] H. Katsura, N. Nagaosa, and A. V. Balatsky, Phys. Rev. Lett. 95, 057205 (2005).

[85] J. Vahedi and S. Mahdavifar, Eur. Phys. J. B 85, 171 (2012).

[86] M. Brockmann, A. Klümper, and V. Ohanyan, Phys. Rev. B 87, 054407 (2013).

[87] M. Enderle, C. Mukherjee, B. Fåk, R. K. Kremer, J.-M. Broto, H. Rosner, S.-L. Drechsler, J. Richter, J. Malek, A. Prokofiev, W. Assmus, S. Pujol, J.-L. Raggazzoni, H. Rakoto, M. Rheinstädter, and H. M. Rønnow, Europhys. Lett. 70, 237 (2005).

[88] Y. Naito, K. Sato, Y. Yasui, Y. Kobayashi, Y. Kobayashi, and M. Sato, J. Phys. Soc. Jpn. 76, 023708 (2007).

[89] S. Furukawa, M. Sato, and S. Onoda, Phys. Rev. Lett. 105, 257205 (2010).

[90] Z. Seidov, T. P. Gavrilova, R. M. Eremina, L. E. Svistov, A. A. Bush, A. Loidl, and H.-A. Krug von Nidda, Phys. Rev. B 95, 224411 (2017).

[91] M. Chen and C. D. Hu, Phys. Rev. B 84, 094433 (2011).

[92] Y. Yasui, M. Sato, and I. Terasaki, J. Phys. Soc. Jpn 80, 033707 (2011).

[93] A. Zorko, S. Nellutla, J. van Tol, L. C. Brunel, F. Bert, F. Duc, J.-C. Trombe, M. A. de Vries, A. Harrison, and P. Mendels, Phys. Rev. Lett. 101, 026405 (2008).

[94] I. Rousochatzakis, S. R. Manmana, A. M. Läuchli, B. Normand, and F. Mila, Phys. Rev. B 79, 214415 (2009).

[95] C.-Y. Lee, B. Normand, and Y.-J. Kao, Phys. Rev. B 98, 224414 (2018).

[96] R. Coldea, D. A. Tennant, K. Habicht, P. Smeibidl, C. Wolters, and Z. Tylczynski, Phys. Rev. Lett. 88, 137203 (2002). 